\title{
A SURVEY OF CONSTRAINED GAUSSIAN PROCESS REGRESSION: APPROACHES AND IMPLEMENTATION CHALLENGES
}

\section{Laura Swiler, ${ }^{1, *}$ Mamikon Gulian, ${ }^{1}$ Ari Frankel, ${ }^{2}$ Cosmin Safta, ${ }^{2}$ E John Jakeman ${ }^{1}$}

${ }^{1}$ Center for Computing Research, Sandia National Laboratories, Albuquerque, NM, 87123

${ }^{2}$ Computational Science and Analysis, Sandia National Laboratories, Livermore, $C A, 94550$

*Address all correspondence to: Laura Swiler, Center for Computing Research, Sandia National Laboratories, Albuquerque, NM, 87123, E-mail: lpswile@sandia.gov

Original Manuscript Submitted: 6/15/2020; Final Draft Received: 9/23/2020

\begin{abstract}
Gaussian process regression is a popular Bayesian framework for surrogate modeling of expensive data sources. As part of a broader effort in scientific machine learning, many recent works have incorporated physical constraints or other a priori information within Gaussian process regression to supplement limited data and regularize the behavior of the model. We provide an overview and survey of several classes of Gaussian process constraints, including positivity or bound constraints, monotonicity and convexity constraints, differential equation constraints provided by linear PDEs, and boundary condition constraints. We compare the strategies behind each approach as well as the differences in implementation, concluding with a discussion of the computational challenges introduced by constraints.
\end{abstract}

KEY WORDS: constrained Gaussian process, scientific machine learning, physicsconstrained machine learning, physics-informed machine learning

\section{INTRODUCTION}

There has been a tremendous surge in the development and application of machine learning models in recent years, due to their flexibility and capability to represent trends in complex systems (Hastie et al., 2016). The parameters of a machine learning model can often be calibrated, with sufficient data, to give high fidelity representations of the underlying process (Frankel et al., 2019b,c; Jones et al., 2018; Raissi et al., 2017). It is now feasible to construct deep learning models over datasets of tens of thousands to millions of data points with modern computational resources (Dean et al., 2012). In many scientific applications, however, there may not be large amounts of data available for training. Unlike data from internet or text searches, computational and physical experiments are typically extremely expensive. Moreover, even if ample data exists, the machine learning model may yield behaviors that are inconsistent with what is expected physically when queried in an extrapolatory regime.

To aid and improve the process of building machine learning models for scientific applications, it is desirable to have a framework that allows the incorporation of physical principles 
and other a priori information to supplement the limited data and regularize the behavior of the model. Such a framework is often referred to as "physics-constrained" machine learning within the scientific computing community (Brunton et al., 2016; Jones et al., 2018; Lee and Carlberg, 2018; Ling et al., 2016; Lusch et al., 2018; Pan and Duraisamy, 2018; Raissi and Karniadakis, 2018). Karpatne et al. (2017) provide a taxonomy for theory-guided data science, with the goal of incorporating scientific consistency in the learning of generalizable models. The information used to constrain models can be simple, such as known range or positivity constraints, shape constraints, or monotonicity constraints that the machine learning model must satisfy. The constraints can also be more complex; for example, they can encode knowledge of the underlying data-generating process in the form of a partial differential equation. Several recent conferences highlight the interest in "physics-informed" machine learning (Berkeley Institute for Data Science, 2019; Los Alamos Center for Nonlinear Studies, 2020; Microsoft, 2019; Stanford University, 2020; University of Washington, 2019).

Much of the existing research in physics-informed machine learning has focused on incorporating constraints in neural networks (Jones et al., 2018; Ling et al., 2016), often through the use of objective/loss functions, which penalize constraint violation (Cyr et al., 2019; Magiera et al., 2019; Mao et al., 2020; Raissi, 2018; Raissi et al., 2019). Other works have focused on incorporating prior knowledge using Bayesian inference that expresses the data-generating process as dependent on a set of parameters, the initial distribution of which is determined by the available information, e.g., functional constraints (Jidling et al., 2017; Wang and Berger, 2016). Unlike deterministic learning approaches, the predictions made using approximations trained with Bayesian inference are accompanied with probabilistic estimates of uncertainty/error.

Within the Bayesian regression framework, Gaussian processes (GPs) are popular for constructing "surrogates" or "emulators" of data sources that are very expensive to query. The use of GPs in a regression framework to predict a set of function values is called Gaussian process regression (GPR). An accurate GPR can often be constructed using only a relatively small number of training data (e.g., tens to hundreds), which consists of pairs of input parameters and corresponding response values. Once constructed, the GPR can be thought of as a machinelearned metamodel and used to provide fast, cheap function evaluations for the purposes of prediction, sensitivity analysis, uncertainty quantification, calibration, and optimization. GP regression models are constructed with data obtained from computational simulation (Gramacy, 2020) or field data; in geostatistics, the process of applying Gaussian processes to field data has been used for decades and is frequently referred to as kriging (Chilès and Desassis, 2018).

In this survey we focus on the use of constrained GPRs that honor or incorporate a wide variety of physical constraints (Bachoc et al., 2019; Da Veiga and Marrel, 2012; Jensen et al., 2013; López-Lopera et al., 2018; Raissi et al., 2017; Riihimäki and Vehtari, 2010; Solak et al., 2003; Yang et al., 2018). Specifically, we focus on the following topics, after a short review of Gaussian process regression in Section 2. Section 3 presents an overview and a classification of constraints according to how the constraint is enforced during the construction of a GP. Section 4 discusses bound constraints, in which the GP prediction may be required to be positive, for example, or the prediction may be required to fall between upper and lower bounds. Section 5 discusses monotonicity and related convexity constraints. Constraints may also be more tightly integrated with the underlying physics: the GP can be constrained to satisfy linear operator constraints, which represent physical laws expressed as partial differential equations (PDE). This is discussed in Section 6. Section 7 discusses intrinsic boundary condition constraints. We review several different approaches for enforcing each of these constraint types. Finally, Section 8 is a compendium of computational details for implementing the constraints of Sections 4-7, together 
with a summary of computational strategies for improving GPR and brief commentary about the challenges of applying these strategies for the constrained GPs considered here.

The taxonomy we present is formulated to enable practitioners to easily query this overview for information on the specific constraint(s) they may be interested in. For approaches that enforce different constraints but have significant overlap in methodology, references are made between sections to the prerequisite subsection where the technical basis of an approach is first discussed in detail. This is done, for example, when discussing spline-based approaches, which are used for both bound constraints in Section 4 and monotonicity constraints in Section 5.

Not all physical constraints can be neatly divided into the categories that we focus on in Sections 4-7. For example, with a view toward computer vision, Salzmann and Urtasun (2010) considered GPR for pose estimation under rigid (constant angle and length) and nonrigid (constant length) constraints between points. They proved that linear equality constraints of the form $A \mathbf{y}=\mathbf{b}$, if satisfied by all the data vectors $\mathbf{y}$, are satisfied by the posterior mean predictor of a GP. Then, at the cost of squaring the input dimension, they translated quadratic length constraints into such linear constraints for pairwise products of the input variables. In another example, Frankel et al. (2019a) applied GPR to predict the behavior of hyperelastic materials in which the stress-stretch constitutive relation naturally exhibits rotational invariance. The rotational invariance was enforced by deriving a finite expansion of the Cauchy stress tensor in powers of the Finger tensor that satisfies the rotational invariance by virtue of its structure, and GPR was performed for the coefficients of the expansion. We mention these examples to illustrate that physical constraints are varied, and in some cases the method to enforce them can depend highly on the specific nature of the constraint.

Even within the selected categories represented by Sections 4-7, the literature on constrained Gaussian processes is extensive and expanding rapidly. Consequently, we cannot provide a complete survey of every instance of constrained GPR. Rather, we strive to discuss main areas of research within the field. The goal is to aid readers in selecting methods appropriate for their applications and enable further exploration of the literature. We present selected implementation details and numerical examples, giving references to the original works for further details. Many of the authors of these works have developed codebases and released them publicly. Finally, we remark that we have adopted consistent notation (established in Section 2) for GPR that does not always follow the notation of the original works exactly.

\section{GAUSSIAN PROCESS REGRESSION}

This section provides an overview of unconstrained Gaussian process regression. As mentioned previously, Gaussian process models, or simply Gaussian processes, are popular because they can be used in a regression framework to approximate complicated nonlinear functions with probabilistic estimates of the uncertainty. Seminal work discussing the use of GPs as surrogate models for computational science and engineering applications include the papers of Sacks et al. (1989) and Santner et al. (2003) and the book by Rasmussen and Williams (2006).

A Gaussian process can be viewed as a distribution over a set of functions. A random draw or sample $f$ from a GP is a realization from the set of admissible functions. Specifically, a Gaussian process is a collection of random variables $\left\{f(\mathbf{x}) \mid \mathbf{x} \in \Omega \subset \mathbb{R}^{d}\right\}$ for which, given any finite set of $N$ inputs $X=\left\{\mathbf{x}_{1}, \mathbf{x}_{2}, \ldots, \mathbf{x}_{N}\right\}, \mathbf{x}_{i} \in \Omega$, the collection $f\left(\mathbf{x}_{1}\right), f\left(\mathbf{x}_{2}\right), \ldots, f\left(\mathbf{x}_{N}\right)$ has a joint multivariate Gaussian distribution. A GP is completely defined by its mean and covariance functions, which generate the mean vectors and covariances matrices of these finite-dimensional multivariate normals. Assumptions such as smoothness of $f$, stationarity, and sparsity are used

Volume 1, Issue 2, 2020 
to construct the mean and covariance of the GP prior, and then Bayes's rule is used to constrain the prior on observational/simulation data.

The prediction $\mathbf{f}=\left[f\left(x_{1}\right), f\left(\mathbf{x}_{2}\right), \ldots f\left(\mathbf{x}_{N}\right)\right]^{\top}$ of a Gaussian process with mean function $m(\mathbf{x})$ and a covariance function $k\left(\mathbf{x}, \mathbf{x}^{\prime}\right)$ is a random variable such that

$$
p(\mathbf{f} \mid X)=\mathcal{N}[\mathbf{f} ; m(X), k(X, X)]
$$

where $m(X)$ denotes the vector $\left[m\left(\mathbf{x}_{1}\right), \ldots, m\left(\mathbf{x}_{N}\right)\right]^{\top}$ and $k(X, X)$ denotes the matrix with entries $\left[k\left(\mathbf{x}_{i}, \mathbf{x}_{j}\right)\right]_{1 \leq i, j \leq N}$. The multivariate normal probability density function $\mathcal{N}(\mathbf{f} ; \mathbf{m}, K)$ with mean vector $\mathbf{m}$ and covariance matrix $K$ has the form

$$
\mathcal{N}(\mathbf{f} ; \mathbf{m}, K)=\frac{1}{(2 \pi)^{N / 2}\left|K^{1 / 2}\right|} \exp \left[-\frac{1}{2}(\mathbf{f}-\mathbf{m})^{\top} K^{-1}(\mathbf{f}-\mathbf{m})\right] .
$$

The covariance kernel function $k$ of a Gaussian process must be symmetric and positive semidefinite. Denoting the individual $d$ components of the vector $\mathbf{x}_{i}$ as $x_{i}^{\ell}$, where $\ell=1, \ldots, d$, the squared exponential kernel

$$
k\left(\mathbf{x}_{i}, \mathbf{x}_{j}\right)=\eta^{2} \exp \left[-\frac{1}{2} \sum_{\ell=1}^{d}\left(\frac{x_{i}^{\ell}-x_{j}^{\ell}}{\rho_{\ell}}\right)^{2}\right], \quad \eta, \rho_{1}, \ldots, \rho_{d} \in \mathbb{R}
$$

is popular, but many other covariance kernels are available. The choice of a covariance kernel can have profound impact on the GP predictions (Duvenaud, 2014; Rasmussen and Williams, 2006), and several approaches to constraining GPs that we survey rely on construction of a covariance kernel specific to the constraint.

The density [Eq. (1)], determined by covariance kernel $k$ and the mean $m$, is referred to as a prior for the GP. If the error or noise relating the actual observations $\mathbf{y}=\left[y\left(\mathbf{x}_{1}\right), y\left(\mathbf{x}_{2}\right), \ldots\right.$, $\left.y\left(\mathbf{x}_{N}\right)\right]^{\top}$ collected at the set of inputs $X=\left\{\mathbf{x}_{i}\right\}_{i=1}^{N}$ to the GP prediction $\mathbf{f}$ is assumed to be Gaussian, then the probability of observing data $\mathbf{y}$, given the GP prior, is given by

$$
p(\mathbf{y} \mid X, \mathbf{f})=\mathcal{N}\left(\mathbf{y} ; \mathbf{f}, \sigma^{2} I_{N}\right), \quad \sigma \in \mathbb{R} .
$$

Here, $I_{N}$ denotes the $N \times N$ identity matrix. The density $p(\mathbf{y} \mid X, \mathbf{f})$ is referred to as the likelihood of the GP, and the Gaussian likelihood [Eq. (4)] is by far the most common. As discussed in Section 4.1.2, specific non-Gaussian likelihood functions can be used to enforce certain types of constraints.

The parameters in the covariance kernel function of a GP are referred to as hyperparameters of the GP. We denote them by $\theta$. For the squared exponential kernel [Eq. (3)], the aggregate vector of hyperparameters is $\theta=\left[\eta, \rho_{1}, \ldots, \rho_{d}, \sigma\right]$, where we have included the likelihood/noise parameter $\sigma$ from Eq. (4) as a hyperparameter. In general, finding the best hyperparameters to fit the data is an important step of GPR, known as training. From now on, we explicitly denote the dependence on $\theta$ of the likelihood $p(\mathbf{y} \mid X, \mathbf{f})$ in Eq. (4) and the prior $p(\mathbf{f} \mid X)$ in Eq. (1), writing these as $p(\mathbf{y} \mid X, \mathbf{f}, \theta)$ and $p(\mathbf{f} \mid X, \theta)$, respectively. The marginal likelihood is given by

$$
p(\mathbf{y} \mid X, \theta)=\int p(\mathbf{y} \mid X, \mathbf{f}, \theta) p(\mathbf{f} \mid X, \theta) d \mathbf{f},
$$

and the log marginal likelihood for a GP with a zero-mean prior ( $m \equiv 0)$ can be written (Murphy, 2012; Rasmussen and Williams, 2006) as

$$
\log p(\mathbf{y} \mid X, \boldsymbol{\theta})=-\frac{1}{2} \mathbf{y}^{\top}\left(K(X, X)+\sigma^{2} I_{N}\right)^{-1} \mathbf{y}-\frac{1}{2} \log \left|K(X, X)+\sigma^{2} I_{N}\right|-\frac{N}{2} \log 2 \pi .
$$


Formula (6), derived from Eqs. (5), (1), and (2), is a function of the hyperparameters $\theta$ present in the kernel $k$, which can be optimized to give the most likely values of the hyperparameters given data. This is known as maximum likelihood estimation (MLE) of the hyperparameters.

Once the hyperparameters of the GPR have been chosen, the posterior of the GPs is given by Bayes' rule,

$$
p(\mathbf{f} \mid X, \mathbf{y}, \boldsymbol{\theta})=\frac{p(\mathbf{f} \mid X, \boldsymbol{\theta}) p(\mathbf{y} \mid X, \mathbf{f}, \boldsymbol{\theta})}{p(\mathbf{y} \mid X, \boldsymbol{\theta})} .
$$

Given the prior $p(\mathbf{f} \mid X, \theta)$ [Eq. (1)] and the Gaussian likelihood $p(\mathbf{y} \mid X, \mathbf{f}, \theta)$ [Eq. (4)], the prediction $f^{*}$ of a GPR at a new point $\mathbf{x}^{*}$ can be calculated (Rasmussen and Williams, 2006) as

$$
p\left(f^{*} \mid \mathbf{y}, X, \mathbf{x}^{*}, \theta\right)=\mathcal{N}\left[\widehat{m}\left(\mathbf{x}^{*}\right), \hat{v}\left(\mathbf{x}^{*}\right)\right]
$$

where

$$
\begin{aligned}
\widehat{m}\left(\mathbf{x}^{*}\right) & =k\left(\mathbf{x}^{*}, X\right)\left[K(X, X)+\sigma^{2} I_{N}\right]^{-1} \mathbf{y}, \\
\hat{v}\left(\mathbf{x}^{*}\right) & =k\left(\mathbf{x}^{*}, \mathbf{x}^{*}\right)-k\left(\mathbf{x}^{*}, X\right)\left[K(X, X)+\sigma^{2} I_{N}\right]^{-1}\left[k\left(\mathbf{x}^{*}, X\right)\right]^{\top} .
\end{aligned}
$$

Note that the mean $\widehat{m}\left(\mathbf{x}^{*}\right)$ of this Gaussian posterior is the mean estimate $\mathbb{E}\left[f\left(\mathbf{x}^{*}\right)\right]$ of the predicted function value $f^{*}$ at $\mathbf{x}^{*}$, and the variance $\hat{v}\left(\mathbf{x}^{*}\right)$ is the estimated prediction variance of the same quantity.

We now preface some of the computational issues of inference in GPR that will be important for the constrained case. First, when the GP likelihood is Gaussian, the posterior [Eq. (8)] is also Gaussian, thus it can be computed exactly, and sampling from the posterior is simple. This is generally not the case when the likelihood is not Gaussian. The same issue arises if the density [Eq. (8)] is directly replaced by a non-Gaussian density in the course of enforcing constraints (by truncation, for example). Next, inversion of $\left[K(X, X)+\sigma^{2} I_{N}\right]$, which scales as $N^{3}$, is an omnipresent issue for inference. This poor scaling to large data is compounded by the fact that increased data tends to rapidly increase the condition number of $K(X, X)$ (see Section 8.3.1). Finally, optimizing the hyperparameters of the GP involves the nonconvex objective function [Eq. (6)]; both this function and its derivatives are potentially costly and unstable to compute for the reasons just mentioned. These issues arise for conventional GPR, but throughout Sections 4-7 we shall see that constraining the GPs can make them more severe. Therefore, we review potential strategies for dealing with them in Section 8.

\section{STRATEGIES FOR CONSTRAINTS}

There are many ways to constrain a Gaussian process model. The difficulty with applying constraints to a GP is that a constraint typically calls for a condition to hold globally - that is, for all points $x$ in a continuous domain-for all realizations or predictions of the process. A priori, this amounts to an infinite set of point constraints for an infinite dimensional sample space of functions. This raises a numerical feasibility issue, which each method circumvents in some way. Some methods relax the global constraints to constraints at a finite set of "virtual" points; others transform the output of the GP to guarantee the predictions satisfy the constraints or construct a sample space of predictions in which every realization satisfies the constraints. This distinction should be kept in mind when surveying constrained GPs. For example, the methods in Sections 4.1, 4.4, and 6.2 enforce constraints globally. The methods in Sections 4.2 and 6.1 enforce the constraint at scattered auxiliary data points, be this a result of introducing virtual data points for constraints, incomplete knowledge, or spatial variability.

Volume 1, Issue 2, 2020 
Strategies for enforcing constraints are apparent from the review of GPR in Section 2, which covers posterior prediction for $\mathbf{f}$, the likelihood function for observations $\mathbf{y}$, the kernel prior $K$, and the data involved in GPR. Some methods, such as the warping method of Section 4.1, simply apply a transformation to the output $\mathbf{f}$ of GPR, so the transformed output satisfies the constraint. This transformation is essentially independent of the other components of GPR. One can instead introduce the constraints at the prediction of $\mathbf{f}$, replacing the density [Eq. (8)] by augmenting the data with a discrete set of virtual points in the domain and predicting $\mathrm{f}$ from the GP, given the data and knowledge that the constraint holds at the virtual points. An example of this is in Section 4.2. Next, the likelihood $p(\mathbf{y} \mid X, \mathbf{f})$ provides another opportunity to enforce constraints. One can replace the Gaussian likelihood [Eq. (4)] with a likelihood function such that constraints are satisfied by $y$ regardless of the output $f$. Hyperparameter optimization provides yet another opportunity in which maximization of the marginal log likelihood [Eq. (6)] is augmented with constraints on the posterior predictions of the GP, as in Section 4.3 .

A different strategy is to design a covariance kernel for the prior [Eq. (1)] of the Gaussian process that enforces the constraint. Several of the methods discussed in this survey involve regression with an appropriate joint GP, defined by the constraint, which uses a "four-block" covariance kernel, incorporating the constraint in some of the blocks. This is the strategy used for the linear PDE constraints in Section 6.1. Such methods are based on derivations of linear transformations of GPs. These types of kernels can be combined with other strategies for constraints, such as for the monotonicity constraints of Section 5.1, which use a four-block covariance kernel (for $\mathbf{f}$ and $\mathbf{f}^{\prime}$ ) within a likelihood approach.

Considering Gaussian processes as distributions over functions, another strategy is to consider a function space defined by a certain representation such that a global constraint can be translated into a finite set of constraints, e.g., on the coefficients of a spline expansion in Sections 4.4 and 5.3. Or a representation can be sought such that every element of the sample space satisfies the constraint before the Gaussian process (the distribution) is even introduced. The latter approach is taken in Sections 6.2 and 7; in these cases, this strategy amounts to deriving a specific kernel function related to the representation.

Finally, data provides an opportunity to constrain Gaussian processes implicitly. Some approaches involve proving that, if the data fed into a GP through the posterior formula [Eq. (7)] satisfies the constraint, then the GP predictions satisfy the constraint-either exactly, as for the linear and quadratic equality constraints of Salzmann and Urtasun (2010), or within a certain error, as in the linear PDE constraints discussed in Section 6.3. These results consider properties of GPs that form the basis of such algorithms.

We note that some of the methods we cover may result in a posterior distribution that is no longer Gaussian, unlike the standard GPR posterior [Eq. (8)]. Thus, such "constrained Gaussian processes" no longer meet the definition of a Gaussian process, rendering the former term a misnomer in this strict sense. Nevertheless, we refer to any method that uses the basic steps of GPR, described in Section 2 as a starting point for a constrained regression algorithm, as providing a constrained Gaussian process.

\section{BOUND CONSTRAINTS}

Bound constraints of the form $a \leq f(\mathbf{x}) \leq b$ over some region of interest arise naturally in many applications. For example, regression over chemical concentration data should enforce that predicted values lie between 0 and 1 (Rider and Simmons, 2018). Bound constraints also 
include, as a special case, nonnegativity constraints $f \geq 0(a=0, b=\infty)$. In this section we present three approaches for enforcing bound constraints.

\subsection{Transformed Output and Likelihood}

The most direct way to impose bound constraints on a Gaussian process involves modifying the output of the regression. One way to do this is to transform the output $f$ of the GP using a "warping" function, which satisfies the bounds. The second way is to replace the Gaussian likelihood [Eq. (4)] with a non-Gaussian likelihood that satisfies the bounds, which is then used to obtain a posterior formula for predicting observations $y$ from $f$. The paper by Jensen et al. (2013) provides an overview and comparison of these two methods; we review this below. For the subsequent discussion, we assume that we have a set of observations $y_{i}$ that satisfy the bound constraint: $a \leq y_{i} \leq b$.

\subsubsection{Warping Functions}

Warping functions are used to transform bounded observations $y_{i}$ to unbounded observations $u_{i}$. The field $u$ together with the observations $u_{i}$ are then treated with a traditional GP model using the steps outlined in Section 2. The probit function, which is the inverse cumulative distribution function of a standard normal random variable: $\Phi^{-1}(\cdot)$, is commonly used as a warping function (Jensen et al., 2013). The probit function transforms bounded values $y \in[0,1]$ to unbounded values $u \in(-\infty, \infty)$ via

$$
u=\Phi^{-1}(y) .
$$

The probit function is popular when $y_{i}$ is uniformly distributed in $[0,1]$ because the transformed values $u_{i}$ will be draws from a standard normal Gaussian with zero mean and unit variance. For a discussion of alternative warping functions, we refer the reader to Snelson et al. (2004).

\subsubsection{Likelihood Formulations}

In addition to using warping functions, bound constraints can also be enforced using nonGaussian likelihood functions $p(\mathbf{y} \mid X, \mathbf{f}, \theta)$ that are constructed to produce GP observations that satisfy the constraints. Given a general non-Gaussian likelihood $p(\mathbf{y} \mid X, \mathbf{f}, \theta)$, the posterior distribution of GPR predictions is given by Eq. (7). Unlike the posterior in Eq. (8), the posterior in this case is no longer guaranteed to be Gaussian. There are a number of parametric distribution functions with finite support that can be used for the likelihood function to constrain the GP model. Jensen et al. (2013) suggest either a truncated Gaussian (see Section 8.1) or a beta distribution scaled appropriately to the interval $[a, b]$. Their results show that the beta distribution generally performs better.

Unlike the warping method of Section 4.1.1, with either a truncated Gaussian likelihood or a beta likelihood, the posterior [Eq. (7)] is not analytically tractable. Jensen et al. (2013) compare two schemes for approximate inference and prediction using bounded likelihood functions: the Laplace approximation and expectation propagation. These approaches both use a multivariate Gaussian approximation of the posterior but solve for the governing posterior distribution in different ways.

Volume 1, Issue 2, 2020 


\subsection{Discrete Constraints Using Truncated Gaussian Distributions}

By noting that a Gaussian process [Eq. (1)] is always trained and evaluated at a finite set of points $X$, global constraints over continuous domain $\Omega$ (such as an interval in one dimension) can be approximated by constraints at a finite set of $N_{c}$ auxiliary or "virtual" points $\mathbf{x}_{i}, \ldots, \mathbf{x}_{N_{c}} \in \Omega$. This approach, introduced by Da Veiga and Marrel (2012), requires constructing an unconstrained GP and then, over the virtual points, transforming this GP to the truncated multivariate normal density $\mathcal{T} \mathcal{N}(\mathbf{z} ; \mu, \Sigma, \mathbf{a}, \mathbf{b})$ as a postprocessing step. The truncated multivariate normal is defined and discussed in detail in Section 8.1.

More specifically, Da Veiga and Marrel (2012) construct an approximation that is conditioned on a truncated multivariate Gaussian distribution at the auxiliary points. We point out how this approach affects the mean posterior predictions of the GP. The unconstrained mean predictor is conditioned on the data $(X, \mathbf{y})$ :

$$
\mathbb{E}\left[f\left(\mathbf{x}^{*}\right) \mid f(X)=\mathbf{y}\right]
$$

This setup is augmented by a fixed, finite set of discrete points $\left\{\mathbf{x}_{i}\right\}_{i=1}^{N_{c}}$, and the predictor [Eq. (11)] is replaced by the predictor

$$
\mathbb{E}\left[f\left(\mathbf{x}^{*}\right) \mid f(X)=\mathbf{y} \quad \text { and } \quad a \leq f\left(\mathbf{x}_{i}\right) \leq b \quad \text { for all } \quad i=1,2, \ldots N_{c}\right]
$$

As $\left[f\left(\mathbf{x}_{1}\right), \ldots, f\left(\mathbf{x}_{N_{c}}\right)\right]^{\top}$ is normally distributed in the unconstrained case [Eq. (11)], in the constrained case [Eq. (12)] it is distributed according to the truncated multivariate normal.

In a few special cases, the mean and covariance of the truncated normal can be derived analytically. In one dimension, the mean at a single prediction point, $z_{i}$, is the unconstrained mean plus a factor that incorporates the change in the probability mass of the Gaussian distribution to reflect the truncation

$$
\mathbb{E}\left(z_{i} \mid a \leq z_{i} \leq b\right)=\mu+\sigma \frac{\phi(\alpha)-\phi(\beta)}{\Phi(\beta)-\Phi(\alpha)},
$$

where $\alpha=(a-\mu) / \sigma, \beta=(b-\mu) / \sigma$, and $\phi$ and $\Phi$ are the probability density function and cumulative density function of a univariate standard normal distribution, respectively. In general, sampling and computing the moments of $\mathcal{T} \mathcal{N}(\mathbf{z} ; \mu, \Sigma, \mathbf{a}, \mathbf{b})$ is computationally demanding. Da Veiga and Marrel (2012) estimate moments empirically using an expensive rejection sampling procedure, based on a modified Gibbs sampler, to generate samples that honor the truncation bounds. We discuss the computational challenge of estimating the moments further in Section 8.1.

In contrast to the warping approach (Section 4.1) or the spline approach (Section 4.4), which maintain a global enforcement of the constraints, the bounds in Eq. (12) can depend on the location: $a_{i} \leq f\left(\mathbf{x}_{i}\right) \leq b_{i}$, representing different bounds in different regions of $I$ [see Section 4 of Da Veiga and Marrel (2012) for an example]. A downside of using the approach described here is that it is unclear how many virtual points $x_{i}$ are needed to approximately constrain the GP globally with a prespecified level of confidence; some studies with increasing $N_{c}$ are presented by Da Veiga and Marrel (2012). However, if the number of points can be chosen adequately, this approach can be used to enforce not only bound constraints but also monotonicity and convexity constraints (Da Veiga and Marrel, 2012); see Section 5 for more details. These types of constraints can also include linear transformations of a Gaussian process (Agrell, 2019). 


\subsection{Constrained Maximum Likelihood Optimization to Enforce Nonnegativity Constraints}

Another option for handling bound constraints is to constrain the optimization of the log marginal likelihood [Eq. (6)], so that hyperparameters are chosen to enforce bounds. Pensoneault et al. (2020) introduced this approach to enforce nonnegativity constraints up to a small probability $0<\epsilon \ll 1$ of violation at a finite set of constraint points $\left\{\mathbf{x}_{i}\right\}_{i=1}^{N_{c}}$,

$$
P\left\{\left[f\left(\mathbf{x}_{i}^{*}\right) \mid y, X, \mathbf{x}_{i}^{*}, \theta\right]<0\right\} \leq \epsilon, \quad i=1,2, \ldots, N_{c} .
$$

For a Gaussian likelihood, the unconstrained posterior $f^{*}$ follows a Gaussian distribution [Eq. (8)], and the probabilistic constraint Eq. (13) can be written in terms of the posterior mean $\widehat{m}(\mathbf{x})$ and posterior standard deviation $s(\mathbf{x})=\sqrt{\hat{v}(\mathbf{x})}$, given by Eq. (9), and probit function $\Phi^{-1}$ (see Section 4.1.1):

$$
\widehat{m}\left(\mathbf{x}_{i}^{*}\right)+\Phi^{-1}(\epsilon) s\left(\mathbf{x}_{i}^{*}\right) \geq 0, \quad i=1,2, \ldots, N_{c} .
$$

Pensoneault et al. (2020) chose $\epsilon=2.3 \%$ so that $\Phi^{-1}(\epsilon)=-2$, i.e., the mean minus two standard deviations is nonnegative. With the condition that $f\left(\mathbf{x}_{j}\right)$ be within $v>0$ of the observations $y_{j}, j=1, \ldots, N$, the maximization of the log marginal likelihood then becomes

$$
\begin{aligned}
\text { Seek } & \theta^{*}=\underset{\theta}{\operatorname{argmax}} \log [p(\mathbf{y} \mid X, \theta)] \\
\text { subject to } & 0 \leq \widehat{m}\left(\mathbf{x}_{i}\right)-2 s\left(\mathbf{x}_{i}\right), \quad i=1, \ldots, N_{c} \\
\text { and } & 0 \leq v-\left|y_{j}-f\left(\mathbf{x}_{j}\right)\right|, \quad j=1, \ldots, N .
\end{aligned}
$$

Pensoneault et al. (2020) solve the constrained optimization problem [Eq. (14)] with $v=0.03$ using a nonlinear interior point solver, demonstrating that nonnegativity is enforced with high probability and also that posterior variance is significantly reduced. While this tends to be more expensive than a usual unconstrained optimization of the marginal log marginal likelihood, the effect on the posterior [Eq. (8)] is to change the hyperparameters, while preserving the Gaussian form, so that more expensive inference methods such as Markov chain Monte Carlo (MCMC) are not required. In principle, two-sided bounds and other types of constraints can be treated in this fashion, although Pensoneault et al. (2020) consider nonnegative constraints in their numerical examples.

\subsection{Splines}

Maatouk and Bay (2017) present a constrained Gaussian process formulation involving splines, where they place a multivariate Gaussian prior on a class of spline functions. The constraints are incorporated through constraints on the coefficients of the spline functions. To avoid the difficulty of enforcing a bound constraint $a \leq f(\mathbf{x}) \leq b$ globally on a continuous domain $\Omega$ for all predictions, the approaches in Sections 4.2 and 4.3 enforced constraints only at a finite set of points. In contrast, the approach taken by Maatouk and Bay (2017) is to instead consider a spline interpolant whose finite set of knot values are governed by a GP. This reduces the infinitedimensional GP to a finite-dimensional one, for which the distributions of the knot values (i.e., the coefficients of the spline expansion) must be inferred. By using a set of piecewise linear splines that form a partition of unity, this approach guarantees that the set of all values between neighboring knots are bounded between the values of the knots. Thus if the knot values satisfy

Volume 1, Issue 2, 2020 
prescribed bound or monotonicity constraints, then so must all values in between them; that is, the global constraints are satisfied if the finite-dimensional constraints are. The problem then reduces to sampling the knot values from a truncated multivariate normal.

\subsubsection{GPR for Spline Coefficients}

We first discuss the spline formulation in one input dimension, and without loss of generality assume that the process being modeled is restricted to the domain $[0,1]$. Let $h(x)$ be the standard tent function, i.e., the piecewise linear spline function defined by

$$
h(x)=\max (1-|x|, 0),
$$

and define the locations of the knots to be $x_{i}=i / M$ for $i=0,1, \ldots, M$, with $M+1$ total spline functions. Then for any set of spline basis coefficients $\xi_{i}$, the function representation is given by

$$
f(x)=\sum_{i=0}^{M} \xi_{i} h\left[M\left(x-x_{i}\right)\right]=\sum_{i=0}^{M} \xi_{i} h_{i}(x) .
$$

This function representation gives a $C^{0}$ piecewise linear interpolant of the point values $\left(x_{i}, \xi_{i}\right)$ for all $i=0,1, \ldots, M$.

The crux of the spline approach to GPR lies in the following argument. Suppose we are given a set of $N$ data points at unique locations $\left(x_{j}, y_{j}\right)$. Define the matrix $A$ such that

$$
A_{i j}=h_{i}\left(x_{j}\right)
$$

Then any set of spline coefficients $\xi$ that satisfy the equation

$$
A \xi=\mathbf{y}
$$

will interpolate the data exactly. Clearly solutions to this system of equations will exist only if the rank of $A$ is greater than $N$, which requires that any given spline basis spans no more than two data points. Intuitively, this is because a linear function is only guaranteed to interpolate two points locally. Supposing that we make $M$ large enough to satisfy this condition, we can find multiple solutions to the system Eq. (15).

We now assume the knot values $\xi$ to be governed by a Gaussian process with covariance function $K$. Because a linear function of a GP is also a GP, the values of $\xi$ and $\mathbf{y}$ are governed jointly (López-Lopera et al., 2018; Maatouk and Bay, 2017) by a GP prior in the form

$$
\left[\begin{array}{l}
\mathbf{y} \\
\xi
\end{array}\right] \sim \mathcal{N}\left(\left[\begin{array}{l}
0 \\
0
\end{array}\right],\left[\begin{array}{cc}
A K A^{\top} & K A^{\top} \\
A K & K
\end{array}\right]\right),
$$

where each entry of the covariance matrix is understood to be a matrix. Upon observation of the data $\mathbf{y}$, the conditional distribution of the knot values subject to $\mathbf{y}=A$ z is given by

$$
p(\xi \mid \mathbf{y}=A \xi)=\mathcal{N}\left(\xi ; K A^{\top}\left(A K A^{\top}\right)^{-1} \mathbf{y}, K-K A^{\top}\left(A K A^{\top}\right)^{-1} A K\right) .
$$

This formula is similar to that proposed by Wilson and Nickisch (2015), in which a GP is interpolated to a regular grid design to take advantage of fast linear algebra. In this case, we are now interested in evaluating the distribution further conditioned on the inequality constraints given by

$$
\begin{aligned}
p(\xi \mid \mathbf{y} & =A \xi, \mathbf{a} \leq \xi \leq \mathbf{b}) \\
& =\mathcal{T} \mathcal{N}\left(\xi ; K A^{\top}\left(A K A^{\top}\right)^{-1} \mathbf{y}, K-K A^{\top}\left(A K A^{\top}\right)^{-1} A K, \mathbf{a}, \mathbf{b}\right),
\end{aligned}
$$


where the truncated normal density $\mathcal{T} \mathcal{N}(\mu, \Sigma, \mathbf{a}, \mathbf{b})$ is defined and discussed in Section 8.1. We illustrate bound constrained GPs using this approach in Fig. 1. We discuss monotonicity constraints using this approach in Section 5.3 and constrained MLE estimation of the hyperparameters in Section 8.2. Several constraint types can be combined in this approach, in which case $\mathcal{C}$ in Eq. (16) is a convex set defined by a set of linear inequalities in $\xi$ (López-Lopera et al., 2018).

\subsubsection{Sampling}

Just as for the discrete constraint method discussed in Section 4.2, sampling from the truncated normal distribution for the spline coefficients $\xi$ introduces a new computational challenge into the GPR framework. While we discuss this in more detail and for several dimensions in Section 8.1, we give a cursory discussion of this following Maatouk and Bay (2017). We consider one dimension and the one-sided constraint $f(x) \geq b$ on $[0,1]$.

The original method of Maatouk and Bay (2017) was to use a rejection sampling approach by sampling from the untruncated distribution with a mean shifted to the mode (or maximum $a$ posteriori point) of the true posterior. That is, one first solves the problem

$$
\xi^{*}=\underset{\xi}{\operatorname{argmin}}(\xi-\mu)^{\top} \Sigma^{-1}(\xi-\mu),
$$

subject to the bound constraints $\xi \geq \mathbf{b}$, where $\mu=K A^{\top}\left(A K A^{\top}\right)^{-1} \mathbf{y}$ and $\Sigma=K-$ $K A^{\top}\left(A K A^{\top}\right)^{-1} A K$. This is a convex quadratic program (assuming the covariance matrix is not too ill-conditioned) and may be solved efficiently. One then draws samples from $\mathcal{N}\left(\xi^{*}, \Sigma\right)$ and accepts or rejects the samples based on an inequality condition, described in more detail in Maatouk and Bay (2016). This is a simple approach, but it does not perform well at larger scale. The probability of rejecting any sample increases exponentially with the number of splines $M$. Furthermore, imprecision in the mode evaluation from the optimization process can lead to a deterioration of acceptance (for example, if the computed mode only satisfies monotonicity constraints to within some solver tolerance). Other approaches to sampling from the multivariate normal rely on Markov chain Monte Carlo methods and are discussed in Section 8.1.
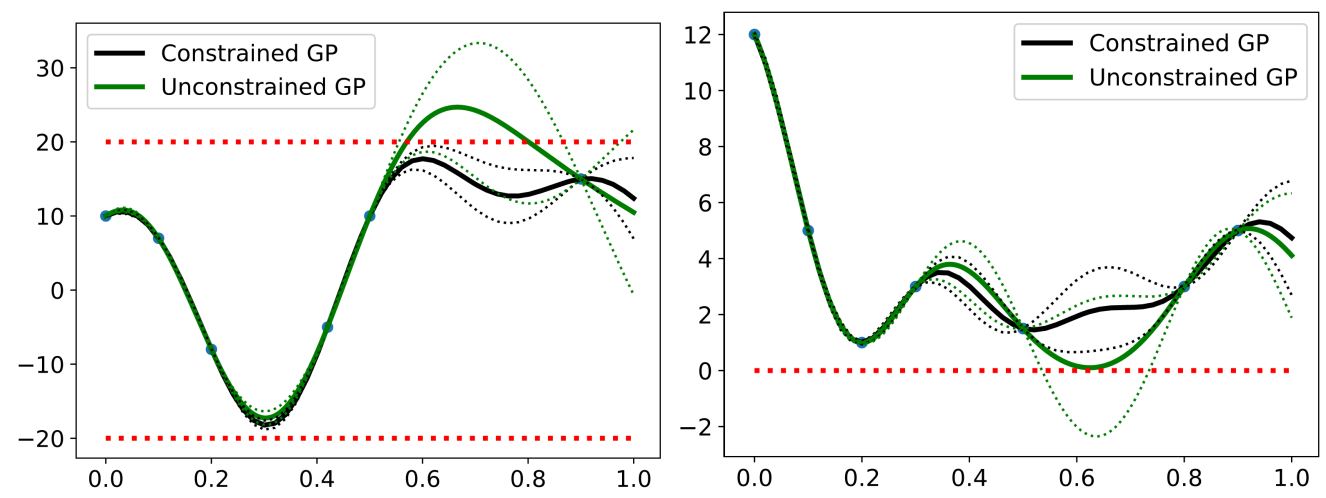

FIG. 1: Left: Comparison of a bound constrained GP with lower bound $a=-20$ and upper bound $b=20$ versus an unconstrained GP. Right: Comparison of a positivity constrained GP (lower bound $a=0$ ) versus an unconstrained GP. The data and hyperparameters are from Maatouk and Bay (2017). The dotted lines are $\mu \pm 1 \sigma$ prediction intervals.

Volume 1, Issue 2, 2020 


\subsubsection{Multidimensional Setting}

The extension of the spline approach to higher dimensions is straightforward. The spline knots must be arranged in a regular grid with $M_{j}+1$ points in each dimension $j$, and the process is restricted to a hypercube domain of size $[0,1]^{d}$ for number of dimensions $d$. Under this restriction, the underlying function may be approximated with the tensor-product spline expansion

$$
f(\mathbf{x})=\sum_{i_{1}=0}^{M_{1}} \sum_{i_{2}=0}^{M_{2}} \ldots \sum_{i_{d}=0}^{M_{d}} \xi_{i_{1}, i_{2}, \ldots, i_{d}} h_{i_{1}, i_{2}, \ldots, i_{d}}\left(x_{1}, x_{2}, \ldots, x_{d}\right) \text {, }
$$

where, with $h_{i}^{j}=h\left[M_{j}\left(x-x_{i_{j}}\right)\right]$,

$$
h_{i_{1}, i_{2}, \ldots, i_{d}}(\mathbf{x})=h_{i_{1}}^{1} \otimes h_{i_{2}}^{2} \otimes \ldots \otimes h_{i_{d}}^{d}(\mathbf{x})=h_{i_{1}}^{1}\left(x_{1}\right) h_{i_{2}}^{2}\left(x_{2}\right) \ldots h_{i_{d}}^{d}\left(x_{d}\right),
$$

for knot locations $\left(x_{i_{1}}, x_{i_{2}}, \ldots, x_{i_{d}}\right)$ and coefficients $\xi_{i_{1}, i_{2}, \ldots, i_{d}}$ for $0 \leq i_{j} \leq M_{j}$. The inference process from this representation proceeds as before, as any set of observed data values can be expressed as $y=A \xi$ for the appropriately defined matrix $A$, and the coefficient values $\xi$ may be inferred with a truncated multivariate normal distribution.

The primary issue with the multidimensional extension is the increase in cost. The spline approach suffers from the curse of dimensionality since the number of spline coefficients that must be inferred scales as $M^{d}$ with $M$ knots per dimension, leading to $O\left(M^{3 d}\right)$ scaling of the inference cost. This cost is further complicated by the fact that the spline formulation requires enough spline coefficients to guarantee interpolation through the data points in all dimensions, which means that $M \geq N$. Some potential methods for addressing computational complexity are discussed later in this work. The need for efficient sampling schemes is also increased in the multidimensional setting, as the acceptance ratio of a simple rejection sampler, as discussed in Section 4.4.2, decreases as the dimensionality (i.e., number of coefficients to infer) increases. This is partially addressed by the Gibbs sampling schemes referred to above, but those schemes also begin to lose efficiency as the size of the problem increases; for other approaches, see Section 8.1.

\section{MONOTONICITY CONSTRAINTS}

Monotonicity constraints are an important class of "shape constraints," which are frequently required in a variety of applications. For example, Maatouk (2017) applied monotonicity-constrained GPR for the output of the Los Alamos National Laboratory "Lady Godiva" nuclear reactor, which is known to be monotonic with respect to the density and radius of the spherical uranium core. Kelly and Rice (1990) considered monotonic Bayesian modeling of medical doseresponse curves, as did Brezger and Steiner (2008), for predicting sales from various prices of consumer goods.

Roughly speaking, given a method to enforce bound constraints, monotonicity constraints can be enforced by utilizing this method to enforce $\mathbf{f}^{\prime} \geq 0$ on the derivative of the Gaussian process in a "co-kriging" setup for the joint GP $\left[\mathbf{f} ; \mathbf{f}^{\prime}\right]$. Indeed, many of the works reviewed in Section 4 considered both bound, monotonicity, and convexity constraints under the general heading of "linear inequality constraints" (Da Veiga and Marrel, 2012; Maatouk and Bay, 2017). As a result, some of the methods below are based on techniques reviewed in Section 4, and we frequently refer to that section. 


\subsection{Constrained Likelihood with Derivative Information}

The work of Riihimäki and Vehtari (2010) enforces monotonicity of a Gaussian process using a probit model for the likelihood of the derivative observations. Probit models are often used in classification problems or binary regression when one wants to predict a probability that a particular sample belongs to a certain class (0 or 1) (Rasmussen and Williams, 2006). Here it is used to generate a probability that the derivative is positive (1) or not (0). Monotonicity is obtained if the derivatives at all the selected points are 1 or 0 .

Using the probit model, the likelihood ${ }^{\dagger}$ for a particular derivative observation is given by

$$
\Phi(z)=\int_{-\infty}^{z} \mathcal{N}(t ; 0,1) d t
$$

where $\mathcal{N}(t ; 0,1)$ is the probability density function of the standard one-dimensional normal distribution [Eq. (2)]. This likelihood is used within an expanded GPR framework that incorporates derivatives and constraints. As part of this formulation, the original $n \times n$ GP covariance matrix, representing the covariance between $n$ data points, is extended to a four-block covariance matrix. The full covariance matrix is composed of matrices involving the covariance between function values, the covariance between derivative values, and the covariance between function values and derivative values.

Following Riihimäki and Vehtari (2010), our goal is to enforce the $d_{i}$ th partial derivative of $f$ at $\mathbf{x}_{i}$ to be nonnegative, i.e.,

$$
\frac{\partial f}{\partial x_{d_{i}}}\left(\mathbf{x}_{i}\right) \geq 0
$$

at a finite set of virtual points $X_{m}=\left\{\mathbf{x}_{i}\right\}_{i=1}^{m}$. Using the shorthand notation

$$
f_{i}^{\prime}=\frac{\partial f}{\partial x_{d_{i}}}\left(\mathbf{x}_{i}\right), \quad \text { and } \quad \mathbf{f}^{\prime}=\left[\frac{\partial f}{\partial x_{d_{1}}}\left(\mathbf{x}_{1}\right) \ldots \frac{\partial f}{\partial x_{d_{m}}}\left(\mathbf{x}_{m}\right)\right]^{\top}=\left[f_{1}^{\prime} \ldots f_{m}^{\prime}\right]^{\top}
$$

and denoting Riihimäki and Vehtari (2010) use the notation of $m_{d_{i}}^{i}$ rather than $y_{i}^{\prime}$ for observations of $\partial f / \partial x_{d_{i}}\left(\mathbf{x}_{i}\right)$. They also use the term "operating points" for the virtual points. an observation of $f_{i}^{\prime}=\partial f / \partial x_{d_{i}}\left(x_{i}\right)$ by $y_{i}^{\prime}$, we can write

$$
p\left(y_{i}^{\prime} \mid f_{i}^{\prime}\right)=\Phi\left(f_{i}^{\prime} \frac{1}{v}\right)
$$

Here $\Phi(z)$ is the cumulative distribution function of the standard normal distribution, and Eq. (18) approaches a step function as $v \rightarrow 0$. Note that the likelihood function in Eq. (18) forces the likelihood to be zero (for nonmonotonicity) or one (for monotonicity) in most cases. Therefore, by including observations of $y_{i}^{\prime}=1$ at the virtual points, the derivative is constrained to be positive. Riihimäki and Vehtari (2010) point out that Eq. (18) is more tolerable of error than a step function and use $v=10^{-6}$ throughout their article.

The joint prior is now given by

$$
p\left(\mathbf{f}, \mathbf{f}^{\prime} \mid X, X_{m}\right)=\mathcal{N}\left(\mathbf{f}_{\text {joint }} \mid 0, K_{\text {joint }}\right),
$$

${ }^{\dagger}$ This particular likelihood is the inverse of the probit function used for warping output in Eq. (10): it maps a value from $(-\infty, \infty)$ to $[0,1]$, representing the probability that the value is in class 1 (which translates to monotonicity for this application).

Volume 1, Issue 2, 2020 
where

$$
\mathbf{f}_{\text {joint }}=\left[\begin{array}{c}
\mathbf{f} \\
\mathbf{f}^{\prime}
\end{array}\right] \quad \text { and } \quad K_{\text {joint }}=\left[\begin{array}{ll}
K_{\mathbf{f}, \mathbf{f}} & K_{\mathbf{f}, \mathbf{f}^{\prime}} \\
K_{\mathbf{f}^{\prime}, \mathbf{f}} & K_{\mathbf{f}^{\prime}, \mathbf{f}^{\prime}}
\end{array}\right] .
$$

Here, the $n \times n$ matrix $K_{\mathbf{f}, \mathbf{f}}$ denotes the standard covariance matrix for the GP $\mathbf{f}$ assembled over the data locations $X: K_{\mathbf{f}, \mathbf{f}}=k(X, X)$, as in Section 2 where $k$ denotes the covariance function of $\mathbf{f}$. The $m \times m$ matrix $K_{\mathbf{f}^{\prime}, \mathbf{f}^{\prime}}$ in Eq. (19) denotes the covariance matrix between the values of the specified partial derivatives of $\mathbf{f}$ at the operational points $X_{m}$ :

$$
\left[K_{\mathbf{f}^{\prime}, \mathbf{f}^{\prime}}\right]_{i j}=\left[\operatorname{cov}\left(f_{i}^{\prime}, f_{j}^{\prime}\right)\right]=\left[\operatorname{cov}\left(\frac{\partial f}{\partial x_{d_{i}}}\left(\mathbf{x}_{i}\right), \frac{\partial f}{\partial x_{d_{j}}}\left(\mathbf{x}_{j}\right)\right)\right], \quad 1 \leq i, \quad j \leq m .
$$

Riihimäki and Vehtari (2010) show that $(\partial f) /\left(\partial x_{d_{i}}\right)$ is a GP with covariance matrix

$$
\frac{\partial}{\partial x_{d_{i}}} \frac{\partial}{\partial x_{d_{j}}^{\prime}} k\left(\mathbf{x}, \mathbf{x}^{\prime}\right)
$$

so that

$$
\left[K_{\mathbf{f}^{\prime}, \mathbf{f}^{\prime}}\right]_{i j}=\frac{\partial^{2} k}{\partial x_{d_{i}} \partial x_{d_{j}}^{\prime}}\left(\mathbf{x}_{i}, \mathbf{x}_{j}^{\prime}\right), \quad 1 \leq i, \quad j \leq m .
$$

This result is a special case of a linear transformation of a GP; see Section 6.1 for more details. By the same general derivation in that section, the matrix $n \times m$ matrix $K_{\mathbf{f}, \mathbf{f}^{\prime}}$ represents the covariance between $\mathbf{f}$ and $\mathbf{f}^{\prime}$ and is given by

$$
\left[K_{\mathbf{f}, \mathbf{f}^{\prime}}\right]_{i j}=\frac{\partial k}{\partial x_{d_{j}}^{\prime}}\left(\mathbf{x}_{i}, \mathbf{x}_{j}^{\prime}\right), \quad 1 \leq i \leq n, \quad 1 \leq j \leq m
$$

and the $m \times n$ matrix $K_{\mathbf{f}^{\prime}, \mathbf{f}}=K_{\mathbf{f}, \mathbf{f}^{\prime}}^{\top}$, representing the covariance between $\mathbf{f}^{\prime}$ and $\mathbf{f}$.

Putting this all together, we have the posterior probability density of the joint distribution incorporating the derivative information

$$
p\left(\mathbf{f}, \mathbf{f}^{\prime} \mid \mathbf{y}, \mathbf{y}^{\prime}\right)=\frac{1}{Z} p\left(\mathbf{f}, \mathbf{f}^{\prime} \mid X, X_{m}\right) p(\mathbf{y} \mid \mathbf{f}) p\left(\mathbf{y}^{\prime} \mid \mathbf{f}^{\prime}\right),
$$

where $1 / Z$ is a normalizing constant. This density is analytically intractable because of the nonGaussian likelihood for the derivative components. Riihimäki and Vehtari (2010) sample this [Eq. (21)] using expectation propagation. We used an MCMC approach to sample the posterior distribution. This approach is illustrated for an example in Fig. 2; this example is particularly challenging because the data is nonmonotonic, but there is a requirement that the GP be monotonic.

We describe the MCMC approach that we used for Eq. (21). As before, $\mathbf{f}^{*}$ and $\mathbf{y}^{*}$ denote the estimates of these quantities at a new prediction point $\mathrm{x}^{*}$.

$$
\begin{gathered}
p\left(\mathbf{y}^{*} \mid \mathbf{x}^{*}, \mathbf{x}, \mathbf{y}\right)=\int p\left(\mathbf{y}^{*} \mid \mathbf{f}^{*}\right) p\left(\mathbf{f}^{*} \mid \mathbf{x}^{*}, \mathbf{x}, \mathbf{y}\right) d \mathbf{f}^{*}, \\
p\left(\mathbf{f}^{*} \mid \mathbf{x}^{*}, \mathbf{x}, \mathbf{y}\right)=\iint p\left(\mathbf{f}^{*} \mid \mathbf{x}^{*}, \mathbf{f}, \mathbf{f}^{\prime}\right) p\left(\mathbf{f}, \mathbf{f}^{\prime} \mid \mathbf{x}, \mathbf{y}\right) d \mathbf{f} d \mathbf{f}^{\prime} .
\end{gathered}
$$



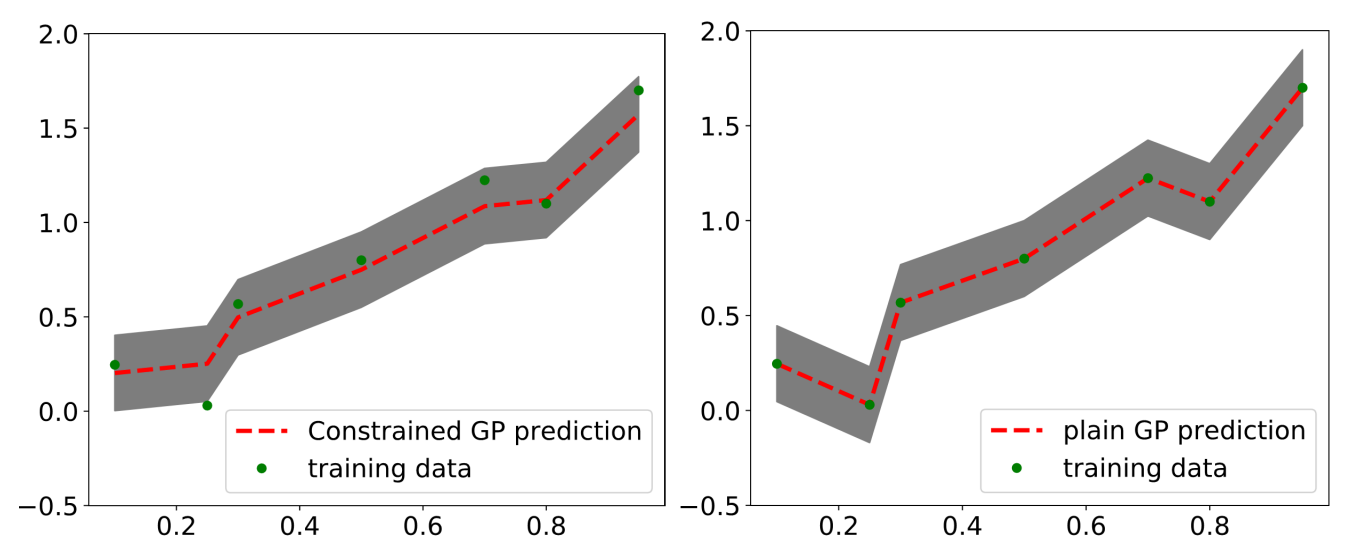

FIG. 2: Comparison of monotonic GP using the constrained likelihood formulation (left) and the unconstrained GP (right). Observations are given at $\{0.1,0.25,0.3,0.5,0.7,0.8,0.95\}$, with monotonicity constraints at the virtual points $\{0.15,0.35,0.55,0.75\}$.

Since $p\left(\mathbf{f}, \mathbf{f}^{\prime} \mid \mathbf{x}, \mathbf{y}\right)$ was computed as samples from MCMC, we can approximate the posterior of $\mathbf{f}^{*}$ as

$$
p\left(\mathbf{f}^{*} \mid \mathbf{x}^{*}, \mathbf{x}, \mathbf{y}\right)=\underset{\mathbf{f}, \mathbf{f}^{\prime} \sim p\left(\mathbf{f}, \mathbf{f}^{\prime} \mid \mathbf{x}, \mathbf{y}\right)}{\mathbb{E}}\left[p\left(\mathbf{f}^{*} \mid \mathbf{x}^{*}, \mathbf{f}, \mathbf{f}^{\prime}\right)\right] \approx \frac{1}{N} \sum_{i=1}^{N} p\left(\mathbf{f}^{*} \mid f_{i}, f_{i}^{\prime}\right) .
$$

The MCMC samples outlined in Eq. (22) are generated over the vector $\left[\mathbf{f} ; \mathbf{f}^{\prime}\right]$. This could be a large vector, indicating a large latent function space, which may pose a challenge to MCMC. Our experience indicates that one must start the MCMC sampling at a good initial point, one that is obtained either by finding the maximum a posteriori point (MAP) or by using a surrogate or interpolation to find a feasible initial point for $\left[\mathbf{f} ; \mathbf{f}^{\prime}\right]$.

Note that this approach leads to the question of how to select the number and placement of the operating points $X_{m}$. Riihimäki and Vehtari (2010) point out that grid-based methods suffer from the curse of dimensionality and that a more efficient strategy may be to successively add new operating points to $X_{m}$ by computing where derivatives of the GP are most likely to be negative for the current choice of $X_{m}$. We did not find much discussion of the placement of virtual points for this method or for the discrete constraint method in Section 4.2. The issue of optimal point placement for the virtual points could be addressed with some of the low-rank methods discussed in Section 8.3.

\subsection{Monotonicity Using Truncated Gaussian Distributions}

The approach, discussed in Section 4.2, for bounding Gaussian processes at a finite set of virtual points can naturally be extended to enforce monotonicity constraints. Specifically, by treating the partial derivatives $\partial f / \partial x_{d_{i}}$ as GPs with covariance kernel functions given by Eq. (20), monotonicity constraints of the same form as Eq. (17) can be enforced at a discrete set of $N_{c}$ virtual points, i.e.,

$$
\frac{\partial f}{\partial x_{d_{i}}}\left(\mathbf{x}_{i}\right) \geq 0, \quad i=1, \ldots, N_{c} .
$$

This is done by treating the partial derivatives $\partial f / \partial x_{d_{i}}$ as GPs with covariance kernel functions given by Eq. (20) and using the joint Gaussian process $f_{\text {joint }}$ with covariance matrix $\Sigma$ given by

Volume 1, Issue 2, 2020 
Eq. (19). Then, given data $(X, \mathbf{y})$ for $f$, Da Veiga and Marrel (2012) replace the unconstrained predictor [Eq. (11)] by the predictor

$$
\mathbb{E}\left[f\left(\mathbf{x}^{*}\right) \mid f(X)=\mathbf{y} \quad \text { and } \quad 0 \leq \frac{\partial f}{\partial x_{d_{i}}}\left(\boldsymbol{x}_{i}\right) \quad \text { for all } \quad i=1,2, \ldots N_{c}\right] .
$$

This is analogous to the predictor [Eq. (12)] used for bound constraints. As a postprocessing step, per Eq. (23) the density $\mathcal{N}(\mu, \Sigma)$ for $\mathbf{f}^{\prime}$ over the virtual points $\left\{\mathbf{x}_{i}\right\}_{i=1}^{N_{c}}$ is replaced by the density $\mathcal{T} \mathcal{N}(\mu, \Sigma, 0, \infty)$; this density is discussed more in Section 8.1.

\subsection{Monotonic Splines}

The spline formulation, presented in Section 4.4 to globally enforce a bound constraint of the form $f \geq a$, may be extended easily to enforce monotonicity constraints or other linear inequalities. For example, if $C$ is a first-order (backward or forward) finite difference matrix relating neighboring spline values, then monotonicity is enforced globally by sampling values of the knots $\xi$ subject to the constraint

$$
C \xi \geq 0,
$$

see López-Lopera et al. (2018) or Maatouk and Bay (2017). This inequality is also used in the rejection sampler of Section 4.4.2 as a constraint to identify the MAP estimate to increase the sampling efficiency. Bound and monotonicity constraints can be enforced simultaneously by requiring both $\xi \geq \mathbf{b}$ and $C \xi \geq 0$ in the sampling, though the acceptance ratio drops substantially with combined constraints.

\subsection{Convexity}

The sections above illustrated how a method for bound constraints can be used with first derivatives of a Gaussian process $f$ to enforce $\partial f / \partial x_{i} \geq 0$ and thereby monotonicity for the GP $f$, either globally, as in Section 5.3 or at a finite set of virtual points, as in Sections 5.1 and 5.2. Similar nonnegativity constraints can be applied to higher derivatives of $f$ as well. In one dimension, this can be used to enforce convexity via the constraint

$$
\frac{\partial^{2} f}{\partial x^{2}} \geq 0,
$$

treating the left-hand side as a GP with covariance kernel

$$
\frac{\partial^{4} k}{\partial x^{2} \partial x^{\prime 2}}\left(x, x^{\prime}\right)
$$

Although monotonicity can be enforced in arbitrary dimensions, convexity presents a challenge in dimensions greater than one, since it cannot be expressed as a simple linear inequality involving the derivatives of $f$, as in Eq. (24). As Da Veiga and Marrel (2012) point out, enforcing convexity in higher dimensions requires that Eq. (24) be replaced by the condition that the Hessian of $f$ be positive semidefinite. Sylvester's criterion yields the equivalent condition that each leading principal minor determinant of the Hessian be positive. Such inequality constraints involve polynomials in partial derivatives of $f$. As polynomial functions of GPs are no longer GPs, the bound constraint methods in Section 4 no longer apply. 
While higher dimensional convexity constraints are outside the scope of this survey, several references we have mentioned discuss the implementation of convexity-constrained Gaussian processes in greater detail. Da Veiga and Marrel (2012) discuss how convexity in one dimension of the form Eq. (24) can be enforced at virtual points using the (partially) truncated multinormal, in a way analogous to Section 5.2, while convexity in two dimensions can be enforced using the elliptically truncated multinormal distribution. Maatouk and Bay (2017) and López-Lopera et al. (2018) point out that for the spline basis considered in Section 4.4, convexity in one dimension amounts to requiring that the successive differences of the values at the spline knots are increasing in magnitude, i.e.,

$$
\xi_{k+1}-\xi_{k} \geq \xi_{k}-\xi_{k-1} \text { for all } k .
$$

This is equivalent to requiring that the second-order finite differences be positive. This can also easily be applied in higher dimensions to guarantee that the second partial derivatives are positive globally, although this does not imply convexity.

\section{DIFFERENTIAL EQUATION CONSTRAINTS}

Gaussian processes may be constrained to satisfy linear operator constraints of the form

$$
\mathcal{L} u=f,
$$

given data on $f$ and $u$. When $\mathcal{L}$ is a linear partial differential operator of the form

$$
\mathcal{L}=\sum_{\alpha} C_{\alpha}(\mathbf{x}) \frac{\partial^{\alpha}}{\partial \mathbf{x}^{\alpha}}, \quad \alpha=\left(\alpha_{1}, \ldots, \alpha_{d}\right), \quad \frac{\partial^{\alpha}}{\partial \mathbf{x}^{\alpha}}=\frac{\partial^{\alpha_{1}}}{\partial x_{1}^{\alpha_{1}}} \frac{\partial^{\alpha_{2}}}{\partial x_{2}^{\alpha_{2}}} \ldots \frac{\partial^{\alpha_{d}}}{\partial x_{d}^{\alpha_{d}}}
$$

Eq. (25) can be used to constrain GP predictions to satisfy known physical laws expressed as linear partial differential equations. In this section we survey methods to constraint GPs with PDE constraints of the form Eq. (25).

\subsection{Block Covariance Kernel}

The work of Raissi et al. (2017) introduced a joint GPR approach that uses a four-block covariance kernel, allowing observations of both the solution $u$ and the forcing $f$ to be utilized. The principle behind this approach is that if $u(\mathbf{x})$ is a GP with mean function $m(\mathbf{x})$ and covariance kernel $k\left(\mathbf{x}, \mathbf{x}^{\prime}\right)$,

$$
u \sim \mathcal{G P}\left[m(\mathbf{x}), k\left(\mathbf{x}, \mathbf{x}^{\prime}\right)\right]
$$

and if $m(\cdot)$ and $k\left(\cdot, \mathbf{x}^{\prime}\right)$ belong to the domain of $\mathcal{L}$, then $\mathcal{L}_{\mathbf{x}} \mathcal{L}_{\mathbf{x}^{\prime}} k\left(\mathbf{x}, \mathbf{x}^{\prime}\right)$ defines a valid covariance kernel for a GP with mean function $\mathcal{L}_{\mathbf{x}} m(\mathbf{x})$. This Gaussian process is denoted $\mathcal{L} u$ :

$$
\mathcal{L} u \sim \mathcal{G} \mathcal{P}\left[\mathcal{L}_{\mathbf{x}} m(\mathbf{x}), \mathcal{L}_{\mathbf{x}} \mathcal{L}_{\mathbf{x}^{\prime}} k\left(\mathbf{x}, \mathbf{x}^{\prime}\right)\right]
$$

Note from Eq. (26) that the operator $\mathcal{L}$ takes as input a function of a single variable $\mathbf{x}$. When applying $\mathcal{L}$ to a function of two variables such as $k\left(\mathbf{x}, \mathbf{x}^{\prime}\right)$, we use a subscript as in Eq. (28) to denote the application of $\mathcal{L}$ in the indicated variable, i.e., considering the input to $\mathcal{L}$ as a function of the indicated variable only. Note that a special case of this, for $\mathcal{L}=\partial / \partial x_{d_{i}}$, appeared in Section 5.1. The same formula [Eq. (28)] was utilized in earlier works on GPR with differential equation constraints by Graepel (2003) and Särkkä (2011).

Volume 1, Issue 2, 2020 
The notation $\mathcal{L} u$ for the GP [Eq. (28)] is suggested by noting that if one could apply $\mathcal{L}$ to the samples of the GP $u$, then the mean of the resulting stochastic process $\mathcal{L}[u]$ would indeed be given by

$$
\operatorname{mean}[\mathcal{L}[u](\mathbf{x})]=\mathbb{E}[\mathcal{L}[u](\mathbf{x})]=\mathcal{L} \mathbb{E}[u(\mathbf{x})]=\mathcal{L} m(\mathbf{x}),
$$

and the covariance by

$$
\begin{aligned}
\operatorname{cov}\left[\mathcal{L}[u](\mathbf{x}), \mathcal{L}[u]\left(\mathbf{x}^{\prime}\right)\right] & =\mathbb{E}\left\{\mathcal{L}_{\mathbf{x}}[u(\mathbf{x})] \mathcal{L}_{\mathbf{x}^{\prime}}\left[u\left(\mathbf{x}^{\prime}\right)\right]\right\}=\mathbb{E}\left\{\mathcal{L}_{\mathbf{x}} \mathcal{L}_{\mathbf{x}^{\prime}}\left[u(\mathbf{x}) u\left(\mathbf{x}^{\prime}\right)\right]\right\} \\
& =\mathcal{L}_{\mathbf{x}} \mathbb{E}\left\{\mathcal{L}_{\mathbf{x}^{\prime}}\left[u(\mathbf{x}) u\left(\mathbf{x}^{\prime}\right)\right]\right\}=\mathcal{L}_{\mathbf{x}} \mathcal{L}_{\mathbf{x}^{\prime}} \mathbb{E}\left[u(\mathbf{x}) u\left(\mathbf{x}^{\prime}\right)\right] \\
& =\mathcal{L}_{\mathbf{x}} \mathcal{L}_{\mathbf{x}^{\prime}}\left\{\operatorname{cov}\left[u(\mathbf{x}), u\left(\mathbf{x}^{\prime}\right)\right]\right\}=\mathcal{L}_{\mathbf{x}} \mathcal{L}_{\mathbf{x}^{\prime}} k\left(\mathbf{x}, \mathbf{x}^{\prime}\right) .
\end{aligned}
$$

This justification is formal, as in general the samples of the process $\mathcal{L} u$ defined by Eq. (28) cannot be identified as $\mathcal{L}$ applied to the samples of $u$ (Driscoll, 1973; Kanagawa et al., 2018); a rigorous interpretation involves the posterior predictions and reproducing kernel Hilbert spaces of the processes $u$ and $\mathcal{L} u$ (Berlinet and Thomas-Agnan, 2011; Seeger, 2004).

If scattered measurements $\mathbf{y}_{f}$ on the source term $f$ in Eq. (25) are available at domain points $X_{f}$, then this can be used to train and obtain predictions for $\mathcal{L} u$ from the GP [Eq. (28)] in the standard way. If, in addition, measurements $\mathbf{y}_{u}$ of $u$ are available at domain points $X_{u}$, a GP co-kriging procedure can be used. In this setting, physics knowledge of the form Eq. (25) enters via the data $\left(X_{f}, \mathbf{y}_{f}\right)$ and can be used to improve prediction accuracy and reduce variance of the GPR of $u$. The co-kriging procedure requires forming the joint Gaussian process $[u ; f]$. Similarly to the derivative case considered in Section 5.1, the covariance matrix of the resulting GP is a four block matrix assembled from the covariance matrix of the GP [Eq. (27)] for the solution $u$, the covariance of the GP Eq. (28) for the forcing function, and the cross terms. Given the covariance kernel $k\left(\mathbf{x}, \mathbf{x}^{\prime}\right)$ for $u$, the covariance kernel of this joint GP is

$$
k\left(\left[\begin{array}{c}
\mathbf{x}_{1} \\
\mathbf{x}_{2}
\end{array}\right],\left[\begin{array}{c}
\mathbf{x}_{1}^{\prime} \\
\mathbf{x}_{2}^{\prime}
\end{array}\right]\right)=\left[\begin{array}{rr}
k\left(\mathbf{x}_{1}, \mathbf{x}_{1}^{\prime}\right) & \mathcal{L}_{\mathbf{x}^{\prime}} k\left(\mathbf{x}_{1}, \mathbf{x}_{2}^{\prime}\right) \\
\mathcal{L}_{\mathbf{x}} k\left(\mathbf{x}_{2}, \mathbf{x}_{1}^{\prime}\right) & \mathcal{L}_{\mathbf{x}} \mathcal{L}_{\mathbf{x}^{\prime}} k\left(\mathbf{x}_{2}, \mathbf{x}_{2}^{\prime}\right)
\end{array}\right]=\left[\begin{array}{ll}
K_{11} & K_{12} \\
K_{21} & K_{22}
\end{array}\right] .
$$

The covariance between $u(\mathbf{x})$ and $f\left(\mathbf{x}^{\prime}\right)$ is given by $\mathcal{L}_{\mathbf{x}^{\prime}} k\left(\mathbf{x}_{1}, \mathbf{x}_{2}^{\prime}\right)$ in the upper right block of the kernel and can be justified by a calculation similar to Eq. (29); see Raissi et al. (2017). Similarly, the covariance between $u\left(\mathbf{x}^{\prime}\right)$ and $f(\mathbf{x})$ is represented by the bottom left block $\mathcal{L}_{\mathbf{x}} k\left(\mathbf{x}_{2}, \mathbf{x}_{1}^{\prime}\right)$ of the kernel. In this notation, the joint Gaussian process for $[u ; f]$ is then

$$
\left[\begin{array}{l}
u\left(X_{1}\right) \\
f\left(X_{2}\right)
\end{array}\right] \sim \mathcal{G P}\left(\left[\begin{array}{r}
m\left(X_{1}\right) \\
\mathcal{L} m\left(X_{2}\right)
\end{array}\right],\left[\begin{array}{ll}
K_{11}\left(X_{1}, X_{1}\right) & K_{12}\left(X_{1}, X_{2}\right) \\
K_{21}\left(X_{2}, X_{1}\right) & K_{22}\left(X_{2}, X_{2}\right)
\end{array}\right]\right),
$$

where $K_{12}\left(X_{1}, X_{2}\right)=\left[K_{21}\left(X_{2}, X_{1}\right)\right]^{\top}$.

Given data $\left(X_{u}, \mathbf{y}_{u}\right)$ and $\left(X_{f}, \mathbf{y}_{f}\right)$, the GP kernel hyperparameters may be trained by assembling the four-block covariance matrix in Eq. (31) with $X_{1}=X_{u}, X_{2}=X_{f}$ :

$$
K_{\text {data }}=\left[\begin{array}{ll}
K_{11}\left(X_{u}, X_{u}\right) & K_{12}\left(X_{u}, X_{f}\right) \\
K_{21}\left(X_{f}, X_{u}\right) & K_{22}\left(X_{f}, X_{f}\right)
\end{array}\right]
$$

and minimizing the negative log marginal likelihood

$$
\begin{gathered}
-\log p\left(\mathbf{y}_{u}, \mathbf{y}_{f} \mid X_{u}, X_{f}, \boldsymbol{\theta}\right)=\frac{1}{2}(\mathbf{y}-\mathbf{m})^{\top} K_{\text {data }}^{-1}(\mathbf{y}-\mathbf{m})+\frac{1}{2} \log \left|K_{\text {data }}\right|+\frac{N}{2} \log (2 \pi), \\
\text { with } \mathbf{y}=\left[\begin{array}{c}
\mathbf{y}_{u} \\
\mathbf{y}_{f}
\end{array}\right] \text { and } \mathbf{m}=\left[\begin{array}{r}
m\left(X_{u}\right) \\
\mathcal{L} m\left(X_{f}\right)
\end{array}\right] .
\end{gathered}
$$


In the presence of noise on measurements of $u$ and $f$, a standard approach analogous to the Gaussian likelihood [Eq. (4)] is to introduce two noise hyperparameters $\sigma_{u}$ and $\sigma_{f}$ and replace the four-block covariance matrix [Eq. (32)] with

$$
\left[\begin{array}{ll}
K_{11}\left(X_{u}, X_{u}\right)+\sigma_{u}^{2} I_{N_{u}} & K_{12}\left(X_{u}, X_{f}\right) \\
K_{21}\left(X_{f}, X_{u}\right) & K_{22}\left(X_{f}, X_{f}\right)+\sigma_{f}^{2} I_{N_{f}}
\end{array}\right] .
$$

The inclusion of the additional terms depending on $\sigma_{u}^{2}$ and $\sigma_{f}^{2}$ corresponds to an assumption of uncorrelated white noise on the measurements $Y_{u}$ and $Y_{f}$, i.e.,

$$
Y_{u}=u\left(X_{u}\right)+\epsilon_{u}, \quad Y_{f}=f\left(X_{f}\right)+\epsilon_{f},
$$

with $\epsilon_{u} \sim \mathcal{N}\left(0, \sigma_{u}^{2} I_{N_{u}}\right)$ and independently $\epsilon_{f} \sim \mathcal{N}\left(0, \sigma_{f}^{2} I_{N_{u}}\right)$, given $N_{u}$ data points for $u$ and $N_{f}$ data points for $f$.

The implementation of the constrained Gaussian process kernel [Eq. (30)] for constraints of the form (25) raises several computational problems. The first is the computation of $\mathcal{L}_{\mathrm{x}} k$ and $\mathcal{L}_{\mathbf{x}} \mathcal{L}_{\mathbf{x}^{\prime}} k$. The most ideal scenario is that in which $k$ has an analytical formula and $\mathcal{L}$ is a linear differential operator, so that these expressions may be computed in closed form by hand or with a symbolic computational software such as Mathematica. This was the approach used for the examples in Raissi et al. (2017, 2018), and Raissi and Karniadakis (2018), including for the heat equation, Burgers's equation, Korteweg-de Vries equation, and Navier-Stokes equations. The nonlinear PDEs listed here were treated using an appropriate linearization. An example of $k$ being parametrized by a neural network (which allows derivatives to be computed using backpropagation) was also considered in Raissi et al. (2018) for the Burgers's equation.

Closed form expressions for the covariance kernel [Eq. (30)] greatly simplify the implementation compared to numerical approximation of $\mathcal{L}_{\mathbf{x}} k$ and $\mathcal{L}_{\mathbf{x}} \mathcal{L}_{\mathbf{x}^{\prime}} k$ using finite-differences or series expansions. As the size of the dataset and therefore the size of the covariance matrix [Eq. (30)] increases, our numerical experiments suggest that any numerical errors in the approximation of the action of $\mathcal{L}$ rapidly lead to the ill-conditioning of the covariance matrix. This in turn can lead to artifacts in the predictions or failure of maximum likelihood estimation with the constrained GP. Ill-conditioning can be reduced by adding an ad-hoc regularization on the diagonal of Eq. (30) at the cost of reducing the accuracy of the regression, potentially negating the benefit of the added constraint. For more general constraints of the form Eq. (25), depending on the form of $k$ or $\mathcal{L}$, numerical methods may be unavoidable. For example, in Raissi et al. (2017) and Gulian et al. (2019), fractional-order PDE constraints (amounting to $\mathcal{L}$ being a nonlocal integral operator with singular kernel) were considered. For these constraints, the kernel blocks $\mathcal{L}_{\mathbf{x}} k$ and $\mathcal{L}_{\mathbf{x}} \mathcal{L}_{\mathbf{x}^{\prime}} k$ had no closed formula. To approximate these terms, a series expansion was used in Raissi et al. (2017), and in Gulian et al. (2019) a numerical method was developed involving Fourier space representations of $\mathcal{L}_{\mathbf{x}} k$ and $\mathcal{L}_{\mathbf{x}} \mathcal{L}_{\mathbf{x}^{\prime}} k$ with Gaussian quadrature for Fourier transform inversion.

A second problem is that the formulation [Eq. 31] requires enforcing the constraint [Eq. (25)] at discrete points of $X_{f}$. Therefore, even if we have complete knowledge of the constraining equation [Eq. (25)] and the forcing term $f$, enhancing the GPR for $u$ by including a high number of virtual data points makes inference as well as maximum likelihood estimation computationally expensive and prone to ill-conditioning. In this regard, the computational approaches discussed in Section 8.3, particularly the subset of data approaches in Section 8.3.1, may be helpful. Figure 3 shows an example of a one-dimensional GP with squared exponential kernel

Volume 1, Issue 2, 2020 


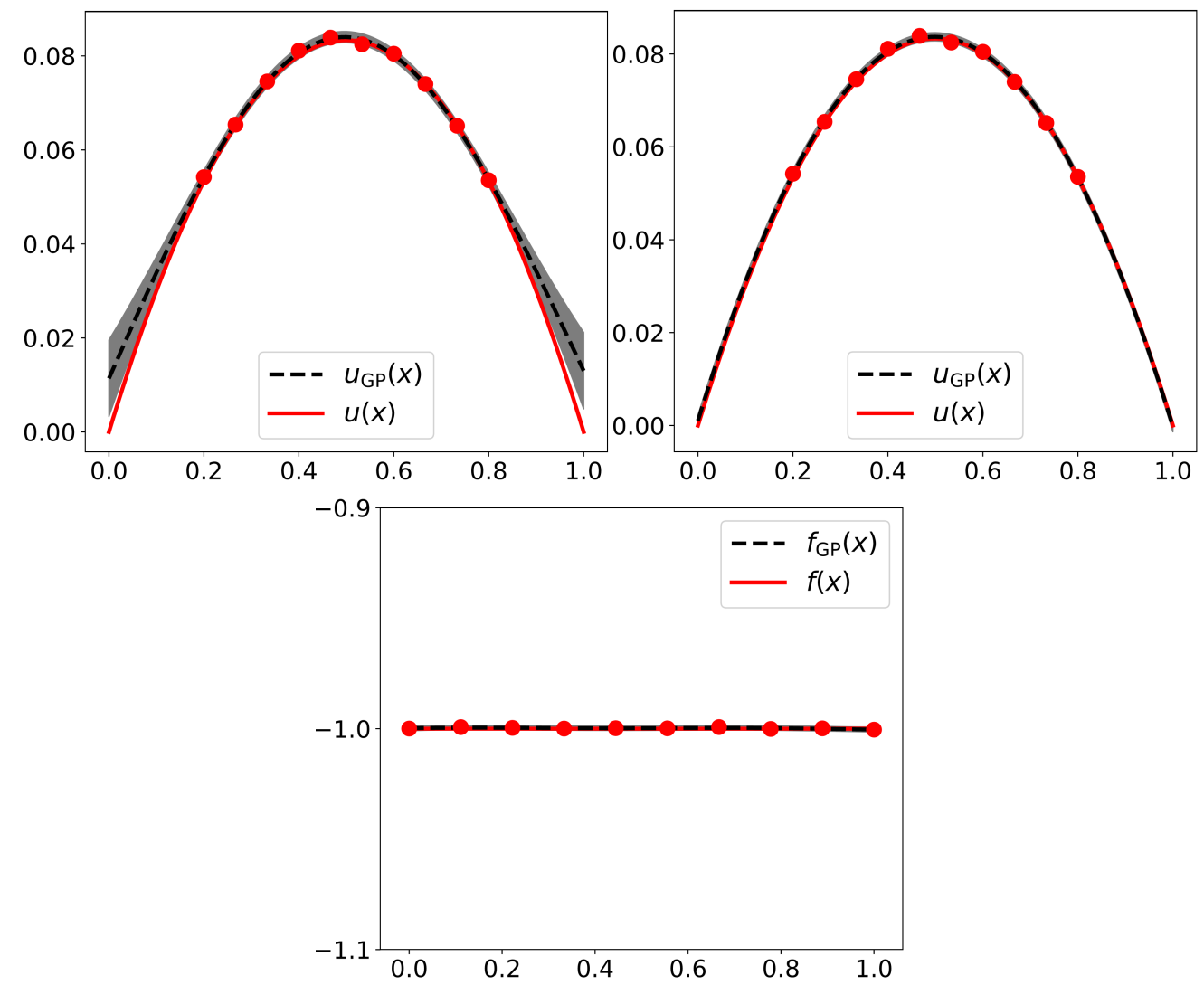

FIG. 3: Comparison of unconstrained and PDE constrained GP. Top-left: Reconstruction of $u$ (red line) with an unconstrained GP (black line) using 10 data points (red dots) in [0.2, 0.8]. Top-right: Reconstruction of $u$ (red solid line) with a PDE-constrained GP (black dotted line) using the same 10 data points (red dots) in $[0.2,0.8]$. Bottom: Right-hand side $f$ of the PDE, with 10 additional data points in $[0,1]$ used for the PDE constraint. Note the improved accuracy of the constrained GP outside $[0.2,0.8]$, due to this constraint data.

constrained to satisfy the differential equation $1=d^{2} u / d x^{2}$ on the interval $[0,1]$. Data is generated from sampling the solution $u=(1 / 8)\left[(2 x-1)^{2}-1\right]$ at 10 points between 0.2 and 0.8 . Both the constrained and unconstrained GPs give a reasonably accurate reconstruction on $[0.2,0.8]$, but the unconstrained GP has poor accuracy outside this subinterval. On the other hand, the constrained GP is augmented by data $f=d^{2} u / d x^{2}=1$ at 10 additional points between 0 and 1 , leading to an improved reconstruction of $u$ outside $[0.2,0.8]$.

\subsection{Transformed Covariance Kernel}

A different approach to constrain Gaussian processes by differential equations is to design a specialized covariance kernel such that the GP satisfies the constraint globally, rather than at a discrete set of auxiliary data points as in Section 6.1. This method dates back to the divergencefree kernel of Narcowich and Ward (1994) for vector-valued GPs. In addition to being a stronger enforcement of the constraint, this method also avoids the computational burden induced by the 
four-block covariance matrix. On the other hand, it has more limited applicability and specialized implementation, as it requires analytically solving for a kernel with the desired constraining property. The authors of Jidling et al. (2017) propose finding a linear operator $\mathcal{G}$, which maps a certain class of functions (modeled by a GP) to the null space of the linear differential operator defining the constraint. The operator $\mathcal{G}$ can then be used to compute the constrained kernel as a transformation of a starting kernel. We now summarize this approach, provide some examples, and compare it in greater detail to other approaches.

Given a linear operator $\mathcal{L}_{\mathrm{x}}$ and a vector-valued GP $\mathrm{f}$, described using a matrix-valued covariance kernel function that encodes the covariance between the entries of the vector $\mathbf{f}$, the constraint

$$
\mathcal{L}_{\mathrm{x}} \mathbf{f}=0
$$

is satisfied if $\mathbf{f}$ can be represented as

$$
\mathbf{f}=\mathcal{G}_{\mathbf{x}} \mathbf{g}
$$

for a transformation $\mathcal{G}_{\mathbf{x}}$ such that

$$
\mathcal{L}_{\mathrm{x}} \mathcal{G}_{\mathbf{x}}=0
$$

In other words, the range of the operator $\mathcal{G}_{\mathrm{x}}$ lies in the nullspace of the operator $\mathcal{L}_{\mathrm{x}}$. Further, provided that $\mathcal{G}_{\mathbf{x}}$ is also a linear operator, if $\mathrm{g}$ is a GP with covariance kernel $k_{\mathrm{g}}$, then by Eq. (34) $\mathrm{f}$ is also a GP with covariance kernel

$$
k_{\mathbf{f}}=\mathcal{G}_{\mathbf{x}} k_{\mathrm{g}} \mathcal{G}_{\mathbf{x}^{\prime}}^{\top} .
$$

Above and throughout this section, we follow Jidling et al. (2017) in using the notation $\mathcal{G}_{\mathbf{x}} k_{\mathbf{g}} \mathcal{G}_{\mathbf{x}^{\prime}}^{\top}$ for the matrix-valued function with $(i, j)$-entry $\sum_{k} \sum_{l}\left[\mathcal{G}_{\mathbf{x}}\right]_{i k}\left[\mathcal{G}_{\mathbf{x}^{\prime}}\right]_{j l}\left[k_{\mathbf{g}}\left(\mathbf{x}, \mathbf{x}^{\prime}\right)\right]_{k l}$; note that if $\mathrm{g}$ and therefore $k_{\mathrm{g}}$ are scalar valued, this reduces to Eq. (29). If the operator [Eq. (35)] can be solved, one can choose a kernel $k_{\mathrm{g}}$ and define a GP f using Eq. (36), which satisfies the constraint [Eq. (33)]. The constraint is satisfied globally by the structure of the covariance kernel; no data is required to enforce it. We refer to this as the transformed covariance kernel approach. Prototypical examples applying the constraint [Eq. (33)] are divergence-free and curl-free constraints on the vector field $\mathbf{f}$; an excellent illustration of such GP vector fields is given by Macêdo and Castro (2008).

As an example of how to apply Eq. (33), consider the enforcement of the curl-free constraint

$$
\mathcal{L}_{\mathbf{x}} f=\nabla \times \mathbf{f}=0,
$$

for a vector field $\mathbf{f}: \mathbb{R}^{3} \rightarrow \mathbb{R}^{3}$. A curl-free vector field can be written $\mathbf{f}=\nabla g$, for a scalar function $g: \mathbb{R}^{3} \rightarrow \mathbb{R}$. So, for $\mathcal{L}_{\mathbf{x}}=\nabla \times$ the choice

$$
\mathcal{G}_{\mathbf{x}}=\nabla, \quad \text { i.e., } \quad \mathcal{G}_{\mathbf{x}} g=\left[\begin{array}{c}
\frac{\partial g}{\partial x_{1}} \\
\frac{\partial g}{\partial x_{2}} \\
\frac{\partial g}{\partial x_{3}}
\end{array}\right]=\left[\begin{array}{c}
\frac{\partial}{\partial x_{1}} \\
\frac{\partial}{\partial x_{2}} \\
\frac{\partial}{\partial x_{3}}
\end{array}\right] g
$$

satisfies Eq. (35). Thus, placing a GP with scalar-valued covariance kernel $k_{g}\left(\mathbf{x}, \mathbf{x}^{\prime}\right)$ on $g$ leads via Eq. (36) to a $3 \times 3$ matrix-valued covariance kernel:

$$
k_{\text {curl-free }}\left(\mathbf{x}, \mathbf{x}^{\prime}\right)=\mathcal{G}_{\mathbf{x}} k_{g} \mathcal{G}_{\mathbf{x}}^{\top}=\left[\begin{array}{ccc}
\frac{\partial^{2}}{\partial x_{1} \partial x_{1}^{\prime}} & \frac{\partial^{2}}{\partial x_{1} \partial x_{2}^{\prime}} & \frac{\partial^{2}}{\partial x_{1} \partial x_{3}^{\prime}} \\
\frac{\partial^{2}}{\partial x_{2} \partial x_{1}^{\prime}} & \frac{\partial^{2}}{\partial x_{2} \partial x_{2}^{\prime}} & \frac{\partial^{2}}{\partial x_{2} \partial x_{3}^{\prime}} \\
\frac{\partial^{2}}{\partial x_{3} \partial x_{1}^{\prime}} & \frac{\partial^{2}}{\partial x_{3} \partial x_{2}^{\prime}} & \frac{\partial^{2}}{\partial x_{3} \partial x_{3}^{\prime}}
\end{array}\right] k_{g}\left(\mathbf{x}, \mathbf{x}^{\prime}\right) .
$$

Volume 1, Issue 2, 2020 
Here, $k_{g}$ is a scalar-valued kernel. If the squared exponential covariance kernel $k_{g}\left(\mathbf{x}, \mathbf{x}^{\prime}\right)=$ $\gamma e^{-\left|\mathbf{x}-\mathbf{x}^{\prime}\right|^{2} /\left(2 \theta^{2}\right)}$ is used, this leads to a closed-form kernel:

$$
k_{\text {curl-free }}\left(\mathbf{x}, \mathbf{x}^{\prime}\right)=\frac{\gamma^{2}}{\theta^{2}} e^{-\frac{\left|\mathbf{x}-\mathbf{x}^{\prime}\right|^{2}}{2 \theta^{2}}}\left[I_{d}-\left(\frac{\mathbf{x}-\mathbf{x}^{\prime}}{\theta}\right)\left(\frac{\mathbf{x}-\mathbf{x}^{\prime}}{\theta}\right)^{\top}\right],
$$

see Jidling et al. (2017) or Jidling (2017). We have derived this for dimension $d=3$, but it is valid in any dimension $d \geq 2$ (Macêdo and Castro, 2008). The specific covariance kernel [Eq. (37)] was introduced by Fuselier (2007) in the context of reproducing kernel Hilbert spaces, and was also used by Baldassarre et al. (2010); Macêdo and Castro (2008); Solin et al. (2018); Wahlström (2015); Wahlström et al. (2013).

In a similar way, one can enforce a divergence-free condition $\nabla \times \mathbf{f}=0$ for a vector-valued GP $\mathbf{f}$ by writing $\mathbf{f}=\nabla \times \mathbf{g}$ and placing a GP prior on a vector field $\mathbf{g}$, as $\nabla(\nabla \times \mathbf{g})=0$ (Jidling et al., 2017). Modeling the components of $g$ as independent and placing a diagonal matrix-valued squared exponential kernel on it leads to divergence-free covariance kernels for $f$ of the form

$$
k_{\text {div-free }}\left(\mathbf{x}, \mathbf{x}^{\prime}\right)=\frac{\gamma^{2}}{\theta^{2}} e^{\frac{\left|\mathbf{x}-\mathbf{x}^{\prime}\right|^{2}}{2 \theta^{2}}}\left\{\left(\frac{\mathbf{x}-\mathbf{x}^{\prime}}{\theta}\right)\left(\frac{\mathbf{x}-\mathbf{x}^{\prime}}{\theta}\right)^{\top}+\left[(d-1)-\frac{\|\mathbf{x}-\mathbf{y}\|^{2}}{\theta^{2}}\right] I_{d}\right\},
$$

see Baldassarre et al. (2010), Jidling (2017), Macêdo and Castro (2008), Wahlström (2015). The specific divergence-free kernel [Eq. (38)] appears to have been introduced by Narcowich and Ward (1994).

In all these examples, solving the key operator equation [Eq. (35)] has been an easy application of vector calculus identities. The work of Jidling et al. (2017) proposes an approach for solving Eq. (35) for general linear constraints involving first-order differential operators, which generalizes the curl-free and divergence-free examples. However, solving Eq. (35) in general is difficult, depends dramatically on $\mathcal{L}$, and may introduce significant computational challenges. For example, to constrain a scalar function on the unit disk $D=\left\{z=\left(x_{1}, x_{2}\right):|z|<1\right\}$ in $\mathbb{R}^{2}$ to satisfy Poisson's equation

$$
\Delta u=\frac{\partial^{2} u}{\partial x_{1}^{2}}+\frac{\partial^{2} u}{\partial x_{2}^{2}}=0 \quad \text { in } \quad D,
$$

i.e., the constraint [Eq. (33)] with $\mathcal{L}_{\mathrm{x}}=\Delta$, one could exploit Poisson's kernel formula

$$
u(r, \theta)=\mathcal{G} g=\frac{1}{2 \pi} \int_{-\pi}^{\pi} P_{r}(\theta-t) g\left(e^{i t}\right) d t,
$$

for a boundary value $g$ defined on $\partial D$. More precisely, $g \in L^{1}(\mathbb{T})$. Thus, one could model $g$ with an appropriate GP with covariance kernel $k_{g}$ and use $\mathcal{G}$ in Eq. (39) to satisfy Eq. (35) and then to define a kernel $k_{u}$ via Eq. (36). However, in this case $G$ is an integral operator, which would make evaluation of Eq. (36) more difficult than the vector calculus examples discussed above. This illustrates that imposing the constraint via solving the operator equation [Eq. (35)] requires using analytical representations of the solution to the constraint equation [Eq. (33)] that vary significantly from case to case. The same issue is illustrated by an example of a biharmonic constraint in one dimension for a scalar GP in Jidling (2017). This is in contrast to the block covariance method of Section 6.1, which involves more straightforward computation of the kernel blocks in Eq. (30). 


\subsection{Empirical Mean and Covariance}

Given an ensemble of realizations of a random field $Y$ on a set of grid points and a smaller set of high-fidelity data on a subset of the low-fidelity grid points, Yang et al. (2018) built a Gaussian process for the unknown field over the unstructured grid that also passes through the high-fidelity data, at the same time ensuring that the GP satisfies the PDE used to generate the low-fidelity ensemble. The ensemble data may be obtained from a large number of simulations over a single unstructured grid of a deterministic solver for a linear PDE, sampling the stochastic parameters in the PDE according to some distribution. The high fidelity may consist of field data obtained through a costly experiment, a situation common in geostatistics.

The idea of the article of Yang et al. (2018) is to compute the mean and covariance function of the GP empirically from these realizations of the random field $Y$. This removes the need to infer the hyperparameters of a covariance function. Instead, one simply calculates the mean and covariance matrix from the random field realizations as follows. Using the notation of Yang et al. (2018), we assume that we have $M$ realizations $Y^{m}(\mathbf{x})$ of the output field $Y(\mathbf{x})$ for $\mathbf{x}$ in the $d$-dimensional grid $\left\{\mathbf{x}_{i}\right\}_{i=1}^{N}$ (the low-fidelity data). Then the mean and covariance kernel are respectively given by

$$
\mu(\mathbf{x}) \approx \mu_{\mathrm{MC}}(\mathbf{x})=\frac{1}{M} \sum_{m=1}^{M} Y^{m}(\mathbf{x})
$$

and

$$
k\left(\mathbf{x}, \mathbf{x}^{\prime}\right) \approx k_{\mathrm{MC}}\left(\mathbf{x}, \mathbf{x}^{\prime}\right)=\frac{1}{M-1} \sum_{m=1}^{M}\left[Y^{m}(\mathbf{x})-\mu_{\mathrm{MC}}(\mathbf{x})\right]\left[Y^{m}\left(\mathbf{x}^{\prime}\right)-\mu_{\mathrm{MC}}\left(\mathbf{x}^{\prime}\right)\right] .
$$

Hence, with $\mathbf{Y}^{m}=\left[Y^{m}\left(\mathbf{x}_{1}\right), \ldots, Y^{m}\left(\mathbf{x}_{N}\right)\right]^{\top}$ and $\mu_{\mathrm{MC}}=\left[\mu_{\mathrm{MC}}\left(\mathbf{x}_{1}\right), \ldots, \mu_{\mathrm{MC}}\left(\mathbf{x}_{N}\right)\right]^{\top}$, the covariance matrix is approximated by

$$
\mathbf{C} \approx \mathbf{C}_{\mathrm{MC}}=\frac{1}{M-1} \sum_{m=1}^{M}\left(\mathbf{Y}^{m}-\boldsymbol{\mu}_{\mathrm{MC}}\right)\left(\mathbf{Y}^{m}-\boldsymbol{\mu}_{\mathrm{MC}}\right)^{\top} .
$$

The above formulas for the mean and covariance of the GP over the unstructured grid $\left\{\mathbf{x}_{i}\right\}_{i=1}^{N}$ can then be used in the usual prediction formula [Eq. (8)] for the posterior mean and variance at any point in the grid, conditioned on high-fidelity data at a subset of points on the grid.

It is important to note that this approach does not assume stationarity of the GP, nor does it assume a specific form of the covariance function. Yang et al. (2018) have shown that physical constraints in the form of a deterministic linear operator are guaranteed to be satisfied within a certain error in the resulting prediction when using this approach. The method was extended to model discrepancy between the low- and high-fidelity data in Yang et al. (2019). They also provide an estimate of the error in preserving the physical constraints. However, as the method uses an empirical mean and covariance, it cannot interpolate for the field between the points where the stochastic realizations are available. The step of GPR for prediction at an arbitrary point $\mathbf{x}^{*}$, represented by Eq. (8), is not available, as the covariance kernel function is bypassed entirely; the covariance is obtained directly in matrix form over the unstructured grid.

\subsection{Specialized Kernel Construction}

Albert and Rath (2020) developed an approach that is suited for GPR with linear PDE constraints of the form Eq. (25) for the case of vanishing or localized source terms $f$. In the latter case, the

Volume 1, Issue 2, 2020 
solution $u$ is represented as a solution to the homogeneous equation $\mathcal{L} u=f$ and an inhomogeneous contribution obtained as a linear model over fundamental solutions corresponding to the point sources.

Focusing on the GPR for the solution $u$ to the homogeneous equation $\mathcal{L} u=0$, a specalized kernel function $k$ is derived from $\mathcal{L}$ such that the GPR prediction satisfies the equation exactly. In this sense, the approach is similar to that of Section 6.2, although the kernel is not obtained from a transformation of a prior kernel but rather is constructed from solutions to the problem $\mathcal{L} u=0$. Albert and Rath (2020) show that such a covariance kernel must satisfy

$$
\mathcal{L}_{\mathbf{x}} k\left(\mathbf{x}, \mathbf{x}^{\prime}\right) \mathcal{L}_{\mathbf{x}^{\prime}}^{\top}=0
$$

in the notation of Section 6.2, and seek kernels $k$ in the form of a Mercer series

$$
k\left(\mathbf{x}, \mathbf{x}^{\prime}\right)=\sum_{i, j} \phi_{i}(\mathbf{x}) \Sigma_{p}^{i, j} \phi_{j}\left(\mathbf{x}^{\prime}\right)
$$

for basis functions $\phi_{i}$ and matrix $\Sigma_{p}$. They point out that convolution kernels can also be considered. Albert and Rath (2020) study the Laplace, heat, and Hemholtz equations, performing MLE and inferring the solution $u$ from the PDE constraint and scattered observations. They construct kernels of the form E41, which satisfy Eq. (40) by selecting $\left\{\phi_{i}\right\}$ to be an orthogonal basis of solution to the corresponding equations. Although the resulting kernels are not stationary and require analytical construction, they result in improved reconstructions of solutions from the observations compared to squared exponential kernels. We note that similar constructions of kernels - as expansions in a suitable basis — are utilized in the approach of Solin and Kok (2019) in the following section to enforce boundary conditions.

\section{BOUNDARY CONDITION CONSTRAINTS}

Boundary conditions and values are yet another type of prior knowledge that may be incorporated into Gaussian process regression. In many experimental setups, measurements can be taken at the boundaries of a system in a cheap and noninvasive way that permits nearly complete knowledge of the boundary values of an unknown field. In other cases, the boundary values may be fully known or controlled by the user, such as for a system in a heat bath. Theoretically, various boundary conditions are often needed to complete the description of a well-posed model. Thus, intrinsic boundary condition constraints on a GP, as opposed to the treatment of boundary measurements as scattered data, may be of interest in applications both for improved accuracy and to avoid the computational burden of an expanded dataset. For one-dimensional GPs, enforcing Dirichlet boundary conditions is trivial; noiseless observations at the boundary can be used to produce a posterior mean and covariance that satisfy the boundary conditions exactly. In higher dimensions, however, it is nontrivial to constrain GPs to satisfy boundary conditions globally over a continuous boundary. Graepel (2003) constructed an example of GPR on the two-dimensional unit square $[0,1]^{2}$ with Dirichlet boundary conditions by writing the solution as a product of a factor represented by a GP and an analytic factor, which was identically zero at the boundary. We discuss a more general approach based on spectral expansions below. 


\subsection{Spectral Expansion Approach}

The work of Solin and Kok (2019) introduced a method based on the spectral expansion of a desired stationary isotropic covariance kernel

$$
k\left(\mathbf{x}, \mathbf{x}^{\prime}\right)=k\left(\left|\mathbf{x}-\mathbf{x}^{\prime}\right|\right),
$$

in eigenfunctions of the Laplacian. For enforcing zero Dirichlet boundary values on a domain $\Omega$, Solin and Kok (2019) use the spectral density (Fourier transform) of the kernel [Eq. (42)],

$$
s(\boldsymbol{\omega})=\int_{\mathbb{R}^{d}} e^{-i \omega \cdot \mathbf{x}} k(|\mathbf{x}|) d \mathbf{x} .
$$

This enters into the approximation of the kernel:

$$
k\left(\mathbf{x}, \mathbf{x}^{\prime}\right) \approx \sum_{\ell=1}^{m} s\left(\lambda_{\ell}\right) \phi_{\ell}(\mathbf{x}) \phi_{\ell}\left(\mathbf{x}^{\prime}\right),
$$

where $\lambda_{j}$ and $\phi_{j}$ are the Dirichlet eigenvalues and eigenfunctions, respectively, of the Laplacian on the domain $\Omega$. In Eq. (44), $s(\cdot)$ is thought of as a function of a scalar variable; since $k$ is isotropic in Eq. (42), so is the Fourier transform $s(\omega)=s(|\omega|)$. Note that the expansion (44) yields a covariance that is zero when $\mathbf{x} \in \partial \Omega$ or $\mathbf{x}^{\prime} \in \partial \Omega$. Thus if the mean of the GP satisfies the zero boundary conditions, Gaussian process predictions using the series (44) will satisfy the boundary condition as well.

\subsection{Implementation}

The first implementation task that presents itself is computation of the Dirichlet spectrum $\left(\lambda_{\ell}, \phi_{\ell}\right)$ of the Laplacian

$$
\begin{aligned}
\Delta \phi_{\ell} & =\lambda_{\ell} \phi_{\ell} \quad \text { in } \quad \Omega, \\
\phi_{\ell} & =0 \quad \text { on } \quad \partial \Omega .
\end{aligned}
$$

For basic domains such as rectangles, cylinders, or spheres, this can be solved in closed form. For general domains, the problem must be discretized and an approximate spectrum computed. Solin and Kok (2019) obtain an approximate spectrum by discretizing the Laplace operator with a finite difference formula and applying a correction factor to the eigenvalues of the resulting matrix. There are many other approaches for computing the spectrum of the Laplacian with various boundary conditions; see, e.g., Song et al. (2017) for an approach using the spectral element method for calculating both Dirichlet and Neumann spectrum in complex geometries. Evaluation of $s\left(\lambda_{\ell}\right)$, where $s$ denotes the spectral density [Eq. (43)] in Eq. (44), is typically not difficult since $s$ is available in closed form for many stationary kernels such as the squared exponential (SE) and Matérn $\left(M_{v}\right)$ kernels:

$$
\begin{aligned}
s_{\mathrm{SE}}(|\omega| ; \gamma, \theta) & =\gamma^{2}\left(2 \pi \theta^{2}\right)^{d / 2} e^{-|\omega|^{2} \theta^{2} / 2} \\
s_{M_{\nu}}(|\omega| ; \gamma, \theta) & =\gamma^{2} \frac{2^{d} \pi^{d / 2}(2 v)^{v} \Gamma(\nu+d / 2)}{\theta^{2} \nu \Gamma(v)}\left(\frac{2 v}{\ell^{2}}+|\omega|^{2}\right)^{(-2 v+d) / 2} .
\end{aligned}
$$

Volume 1, Issue 2, 2020 
Next, we review from Solin and Kok (2019) and Solin and Särkkä (2019) how the formulas for Gaussian processes regression and training can be expressed using the the formulation (44). Given $n$ data points $\left\{\left(\mathbf{x}_{i}, y_{i}\right)\right\}_{i=1}^{n}$, the covariance matrix is approximated using Eq. (44) as

$$
K_{i j}=k\left(\mathbf{x}_{i}, \mathbf{x}_{j}\right) \approx \sum_{\ell=1}^{m} \phi_{\ell}\left(\mathbf{x}_{i}\right) s\left(\lambda_{\ell}\right) \phi_{\ell}\left(\mathbf{x}_{j}\right)
$$

Introducing the $n \times m$ matrix $\Phi$,

$$
\Phi_{i \ell}=\phi_{\ell}\left(\mathbf{x}_{i}\right), \quad 1 \leq i \leq n, \quad 1 \leq \ell \leq m
$$

and the $m \times m$ matrix, $\Lambda=\operatorname{diag}\left(s\left(\lambda_{\ell}\right)\right), 1 \leq \ell \leq m$, can be written as

$$
K \approx \Phi \Lambda \Phi^{\top}
$$

Thus, the covariance matrix $K$ is diagonalized, and for a point $\mathrm{x}^{*}$ we can write the $n \times 1$ vector

$$
\mathbf{k}_{*}=\left[k\left(\mathbf{x}^{*}, \mathbf{x}_{i}\right)\right]_{i=1}^{n} \approx\left[\sum_{\ell=1}^{m} \phi_{\ell}\left(\mathbf{x}_{i}\right) s\left(\lambda_{\ell}\right) \phi_{\ell}\left(\mathbf{x}^{*}\right)\right]_{i=1}^{n}=\Phi \Lambda \mathbf{\Phi}_{*}
$$

where the $m \times 1$ vector $\boldsymbol{\Phi}_{*}$ is defined by

$$
\left[\boldsymbol{\Phi}_{*}\right]_{\ell}=\phi_{\ell}\left(\mathbf{x}^{*}\right), \quad 1 \leq \ell \leq m .
$$

The Woodbury formula can be used to obtain the following expressions for the posterior mean and variance over a point $\mathbf{x}^{*}$, given a Gaussian likelihood $y_{i}=f\left(x_{i}\right)+\epsilon_{i}, \epsilon_{i} \sim \mathcal{N}\left(0, \sigma^{2}\right)$ (Solin and Kok, 2019):

$$
\begin{aligned}
\mathbb{E}\left[f\left(\mathbf{x}^{*}\right)\right] & =\mathbf{k}_{*}^{\top}\left(K+\sigma^{2} I\right)^{-1} \mathbf{y} \\
& =\boldsymbol{\Phi}_{*}^{\top}\left(\Phi^{\top} \Phi+\sigma^{2} \Lambda^{-1}\right)^{-1} \Phi^{\top} \mathbf{y} . \\
\mathbb{V}\left[f\left(\mathbf{x}^{*}\right)\right] & =k\left(\mathbf{x}^{*}, \mathbf{x}^{*}\right)-\mathbf{k}_{*}^{\top}\left(K+\sigma^{2} I\right)^{-1} \mathbf{k}_{*} \\
& =\sigma^{2} \boldsymbol{\Phi}_{*}^{\top}\left(\Phi^{\top} \Phi+\sigma^{2} \Lambda^{-1}\right)^{-1} \mathbf{\Phi}_{*} .
\end{aligned}
$$

Strategies for using this method with non-Gaussian likelihoods are also discussed by Solin and Kok (2019), although we do not go over them here. For use in hyperparameter training, the following formulas were derived in Solin and Särkkä (2019) and Solin and Kok (2019) for the negative log marginal likelihood

$$
\begin{aligned}
-p(y \mid X, \theta) & =\frac{n-m}{2} \log \sigma^{2}+\frac{1}{2} \sum_{\ell=1}^{m} \log \left(\Lambda_{\ell, \ell}\right)+\frac{1}{2} \log \operatorname{det}\left(\sigma^{2} \Lambda^{-1}+\Phi^{\top} \Phi\right) \\
& +\frac{n}{2} \log (2 \pi)+\frac{1}{2 \sigma^{2}}\left[\mathbf{y}^{\top} \mathbf{y}-\mathbf{y}^{\top} \Phi\left(\sigma^{2} \Lambda^{-1}+\Phi^{\top} \Phi\right)^{-1} \Phi^{\top} \mathbf{y}\right]
\end{aligned}
$$


and in Solin and Särkkä (2019) for its derivative:

$$
\begin{aligned}
-\frac{\partial p(y \mid X, \theta)}{\partial \theta_{k}} & =\frac{1}{2} \sum_{\ell=1}^{m} \frac{1}{\Lambda_{\ell, \ell}} \frac{\partial \Lambda_{\ell, \ell}}{\partial \theta_{k}}-\frac{\sigma^{2}}{2} \operatorname{Tr}\left(\left(\sigma^{2} \Lambda^{-1}+\Phi^{\top} \Phi\right)^{-1} \Lambda^{-2} \frac{\partial \Lambda}{\partial \theta_{k}}\right) \\
& -\mathbf{y}^{\top} \Phi\left(\sigma^{2} \Lambda^{-1}+\Phi^{\top} \Phi\right)^{-1}\left(\Lambda^{-2} \frac{\partial \Lambda}{\partial \theta_{k}}\right)\left(\sigma^{2} \Lambda^{-1}+\Phi^{\top} \Phi\right)^{-1} \Phi^{\top} \mathbf{y} \\
-\frac{\partial p(y \mid X, \theta)}{\partial \sigma^{2}} & =\frac{n-m}{2 \sigma^{2}}+\frac{1}{2} \operatorname{Tr}\left(\left(\sigma^{2} \Lambda^{-1}+\Phi^{\top} \Phi\right)^{-1} \Lambda^{-1}\right) \\
& +\frac{1}{2 \sigma^{2}} \mathbf{y}^{\top} \Phi\left(\sigma \Lambda^{-1}+\Phi^{\top} \Phi\right)^{-1} \Lambda^{-1}\left(\sigma \Lambda^{-1}+\Phi^{\top} \Phi\right)^{-1} \Phi^{\top} \mathbf{y} \\
& -\frac{1}{2 \sigma^{4}}\left[\mathbf{y}^{\top} \mathbf{y}-\mathbf{y}^{\top} \Phi\left(\sigma^{2} \Lambda^{-1}+\Phi^{\top} \Phi\right)^{-1} \Phi^{\top} \mathbf{y}\right] .
\end{aligned}
$$

Note that $\Lambda$ is defined by the spectral density $s$ of the kernel $k$, which clearly depends on the kernel hyperparameters $\theta=\left[\theta_{i}\right]$; however, $\Phi$ does not. Typically, derivatives of $\Lambda$ with respect to $\theta_{i}$ can be computed in closed form, which along with formulas (46) and (47) enables accurate first-order optimization of the kernel hyperparameters.

\subsection{Extensions}

The expansion in Solin and Särkkä (2019) was originally developed for the computational advantages of using a low rank approximation to a kernel (see Section 8.3.2 for a discussion of this aspect) rather than for boundary condition constraints. Consequently, the discussions in Solin and Kok (2019) and Solin and Särkkä (2019) focused only on periodic and zero Dirichlet boundary conditions. One possible way to constrain a Gaussian process $f$ to satisfy nonzero Dirichlet conditions would be to write $f=(f-g)+g$, where $g$ is a harmonic function that satisfies a given nonzero Dirichlet condition, and to model $f-g$ as a Gaussian process that satisfies a zero Dirichlet condition using the above approach. Solin and Kok (2019) remark that the method could also be extended to Neumann boundary conditions by using the Neumann eigenfunctions of the Laplacian, although no examples are given. Another limitation is that spectral expansions in Solin and Kok (2019) and Solin and Särkkä (2019) are only considered for isotropic kernels, but they suggest that the approach can be extended to the nonisotropic case.

\section{COMPUTATIONAL CONSIDERATIONS}

In this section, we describe methods that can help reduce the computational cost of constructing constrained GP models. Typically, building a constrained GP is significantly more expensive than training an unconstrained GP because of larger data sets representing derivative constraints, bounds, etc., at virtual points. Consequently, computationally efficient strategies for building constrained GPs are paramount. In Section 8.1 we discuss the truncated multivariate normal distribution, which is a fundamental component of the approaches discussed in Sections 4.1, 4.2, 4.4, and 5.2. We then discuss the related problem of maximum likelihood estimation of the hyperparameters of constrained GPs constructed using the spline approach discussed in Sections 4.4, 5.3, and 5.4. The final Section 8.3 focuses on reducing the numerical linear algebra cost of inference, using low-rank and Kronecker methods, respectively. The majority of approaches surveyed in these two sections were developed for unconstrained GPs; however, some methods have been applied in the constrained setting. Since such numerical recipes are the focus of much

Volume 1, Issue 2, 2020 
deeper survey articles such as Quiñonero-Candela and Rasmussen (2005a); Quiñonero-Candela and Rasmussen (2005b), we have intentionally kept our discussion short, while providing references to applications in constrained GPR where available.

\subsection{The Truncated Multivariate Normal Distribution}

Given a positive-definite covariance matrix $\Sigma$ and vectors $\mathbf{a}, \mathbf{b} \in \mathbb{R}^{d}$ defining a rectangle $\{\mathbf{a} \leq$ $\mathbf{x} \leq \mathbf{b}\}$, the truncated normal distribution is the conditional distribution of the random variable $x \sim \mathcal{N}(\mu, \Sigma)$, given $\mathbf{a} \leq \mathbf{x} \leq \mathbf{b}$. The density $\mathcal{T N}(\mu, \Sigma, \mathbf{a}, \mathbf{b})$ of the truncated normal can be expressed as

$$
\mathcal{T N}(\mathbf{x}, \mu, \Sigma, \mathbf{a}, \mathbf{b})=\frac{1_{\mathbf{a} \leq \mathbf{x} \leq \mathbf{b}}(x)}{C} \mathcal{N}(\mathbf{x} ; \mu, \Sigma),
$$

where the normalization constant

$$
\begin{aligned}
C & =\int_{a_{1}}^{b_{1}} \int_{a_{2}}^{b_{2}} \ldots \int_{a_{d}}^{b_{d}} \mathcal{N}(\mathbf{x} ; \mu, \Sigma) d \mathbf{x}_{1} d \mathbf{x}_{2} \ldots d \mathbf{x}_{d} \\
& =\frac{1}{(2 \pi)^{\frac{d}{2}}|\Sigma|^{\frac{1}{2}}} \int_{a_{1}}^{b_{1}} \int_{a_{2}}^{b_{2}} \ldots \int_{a_{d}}^{b_{d}} \exp \left(-\frac{1}{2}(\mathbf{x}-\mu)^{\top} \Sigma^{-1}(\mathbf{x}-\mu)\right) d \mathbf{x}_{1} d \mathbf{x}_{2} \ldots d \mathbf{x}_{d}
\end{aligned}
$$

is the probability that a sample of $\mathcal{N}(\mu, \Sigma)$ lies in $\{\mathbf{a} \leq \mathbf{x} \leq \mathbf{b}\}$.

For general $\Sigma$ and dimension $d$, computing the normalization constant and sampling from the truncated multinormal distribution [Eq. (48)] can be difficult and require specialized methods. Of course, from the definition Eq. (49) these two problems are related. However, they appear in two different contexts. Calculating integrals of the form Eq. (49), known as Gaussian orthant probabilities, is called for in constrained maximum likelihood estimation of the GPR hyperparameters, while sampling Eq. (48) is needed for posterior prediction in several approaches discussed above. Therefore, we discuss sampling first and discuss evaluation of Gaussian orthant probabilities in the next Section 8.2.

While there are several possible approaches to sampling from Eq. (48), simple Monte Carlo methods scale poorly to high dimensions. One such example-rejection sampling from the mode-was discussed in Section 4.4.2. In principle, it is possible to use a Metropolis-Hastings approach to sample the values of the knots, but it is expected that the dimensionality of the chain for a large number of splines is likely to slow down the convergence of the chain. Several Markov chain Monte Carlo (MCMC) methods were studied by López-Lopera et al. (2018) for sampling the truncated multivariate normal posterior distribution that arises in the spline approach described in Section 4.4. Comparison of expected sample size metrics suggested that Hamiltonian Monte Carlo (HMC) is the most efficient sampler in the setting of that article. A different approach for sampling Eq. (48), based upon elliptical slice sampling and the fast Fourier transform, was presented in Ray et al. (2019).

\subsection{Constrained Maximum Likelihood Estimation for Splines}

We review the work of López-Lopera et al. (2018) which discusses the maximum likelihood estimation of hyperparameters within the spline approach discussed in Sections 4.4 and 5.3. The starting point is the constrained log marginal likelihood function given the constraints $\xi \in \mathcal{C}$, 
where we have denoted $\mathcal{C}=\{\mathbf{a} \leq \xi \leq \mathbf{b}\}$. This is based on the posterior density $p_{\theta}(\mathbf{y} \mid \xi \in \mathcal{C})$ of $\mathbf{y}$ given the constraint $\xi \in \mathcal{C}$, which by Bayes's rule can be expressed as

$$
p_{\theta}(\mathbf{y} \mid \xi \in \mathcal{C})=\frac{p_{\theta}(\mathbf{y}) P_{\theta}(\xi \in \mathcal{C} \mid \Phi \xi=\mathbf{y})}{P_{\theta}(\xi \in \mathcal{C})} .
$$

Taking the logarithm yields a constrained log marginal likelihood function:

$$
\begin{aligned}
\mathcal{L}_{\mathrm{cMLE}} & =\log p_{\theta}(\mathbf{y} \mid \xi \in \mathcal{C}) \\
& =\log p_{\theta}(\mathbf{y})+\log P_{\theta}(\xi \in \mathcal{C} \mid \Phi \xi=\mathbf{y})-\log P_{\theta}(\xi \in \mathcal{C}) \\
& =\mathcal{L}_{\mathrm{MLE}}+\log P_{\theta}(\xi \in \mathcal{C} \mid \Phi \xi=\mathbf{y})-\log P_{\theta}(\xi \in \mathcal{C}) .
\end{aligned}
$$

In the first term, $p_{\theta}(\mathbf{y})$ refers to the probability density function of the random variable $\mathbf{y}$ with hyperparameters $\theta$; thus, the first term is simply the unconstrained log marginal likelihood [Eq. (6)], which we denote $\mathcal{L}_{\mathrm{MLE}}$. In the second and third terms, $P_{\theta}$ refers to the probability of the indicated events. As $\xi$ and $\xi \mid\{\Phi \xi=\mathbf{y}\}$ are both normally distributed by equations Eqs. (1) and (8), respectively, the two last terms in Eq. (50) can be expressed as integrals of a normal density over $\mathcal{C}$, just like the normalization constant Eq. (49). Such integrals can be reduced to integrals over orthants, so the last two terms in Eq. (50) are referred to in López-Lopera et al. (2018) as Gaussian orthant probabilities.

Unlike the sampling of Eq. (48), for which computing such integrals can be avoided with MCMC, calculation of Gaussian orthant probabilities is unavoidable if the user wants to train the kernel hyperparameters using the constrained objective function [Eq. (50)], which we refer to as cMLE. A thorough discussion of numerical approaches to truncated Gaussian integrals is found in Genz and Bretz (2009). López-Lopera et al. (2018) utilize the minimax exponential tilting method of Botev (2017), reported to be feasible for quadrature of Gaussian integrals in dimensions as high as 100, to compute the Gaussian orthant probabilities in Eq. (50) and compare cMLE with MLE. Another current drawback of cMLE is that the gradient of $\mathcal{L}_{\text {cMLE }}$ is not available in closed form, unlike the gradient of $\mathcal{L}_{\text {MLE }}$ (Rasmussen and Williams, 2006). Thus, in López-Lopera et al. (2018), MLE was performed using a limited-memory version of the Broyden-Fletcher-Goldfarb-Shanno algorithm (L-BFGS) optimizer, while cMLE was performed using the method of moving asymptotes. This involved a numerical approximation to the gradient of $\mathcal{L}_{\mathrm{cMLE}}$, which in our experience can impact the accuracy of the optimization. Although these numerical differences hamper direct comparison of MLE and CMLE, it was found by López-Lopera et al. (2018) that for the case of limited data, cMLE can provide more accurate estimation of hyperparameter values and confidence intervals than MLE.

López-Lopera et al. (2018) also studied under which conditions MLE and cMLE yield consistent predictions of certain hyperparameters. This was further studied in Bachoc et al. (2019), in which the authors perform an analysis of MLE and cMLE for the case of fixed-domain asymptotics, i.e., data in a fixed domain, as the number of data points tends to infinity. In this regime of dense data, the effect of constraints is expected to diminish. The authors show that MLE and cMLE yield consistent hyperparameters in this limit for the case of boundedness, monotonicity, and convexity constraints, and suggest quantitative tests to determine if the number of data points is sufficient to suggest unconstrained MLE as opposed to the more expensive cMLE.

\subsection{Scalable Inference}

As pointed out in Section 2, inference in GPR using the entire training dataset (of size $N$ ) scales as $O\left(N^{3}\right)$ due to covariance matrix inversion. This is exacerbated by certain methods to enforce

Volume 1, Issue 2, 2020 
constraints, such as the linear PDE constraints in Section 6.1, which require the inclusion of "virtual" constraint points in the training data. There have been few studies on improving scalability of constrained GPs. Thus, in this section, we mention several promising approaches and possible applications to constrained GPs. Some strategies, including the subset of data approach, the inducing point approach, and the spectral expansion approach, are specific to covariance matrices of GPs. Other methods are based on general linear algebra techniques.

\subsubsection{Subset of Data and Inducing Point Methods}

One notable feature of increasing the density of training data is that the covariance matrix tends to become more ill-conditioned, the result of partially redundant information being added to the matrix. In such situations it is worthwhile to identify a subset of data that minimizes prediction error subject to a maximum dataset size constraint (Quiñonero-Candela and Rasmussen, 2005b). Greedy methods involve sequentially choosing points in the domain that have the maximal predictive variance in order to reduce the uncertainty in the final GP. This choice is natural and has connections to information-theoretic metrics; other metrics include cross-validation prediction error, the likelihood value, or Bayesian mean-square prediction error. Rather than building a new covariance matrix and inverting it for each added point, one may take advantage of the Woodbury matrix inversion lemma and block matrix inversions to efficiently compute the inverse of the covariance matrix (Quiñonero-Candela and Rasmussen, 2005b).

Other methods for performing subset selection are based on local approximation. Frequently, the function values far away from a point of interest may have little influence on the function value there. A simple strategy based on this idea is to select the nearest neighbors to the target point to form the prediction. The local approximation GP approach (Gramacy, 2016; Gramacy and Apley, 2015) combines such local approximation with a greedy search heuristic to identify a better set of points to minimize the mean-squared prediction error at the location of interest.

Using a subset of points to form the GP corresponds to selecting a subset of the rows/columns of a full covariance matrix to represent the dataset. Quiñonero-Candela and Rasmussen (2005a) generalize this to a broad set of low-rank approximations to the full covariance matrix based on inducing points. In these methods, a subset (size $m$ ) of the data is used to form an approximate likelihood or prior for the entire dataset; all of the data is used, but most of the data is modeled as being conditionally dependent on a few inducing points. This reduces the cost of inference from $O\left(N^{3}\right)$ to $O\left(N m^{2}\right)$. The greedy methods discussed above may be applied to identify an optimal set of inducing points.

Such methods may be especially helpful for selection and placement of virtual points for enforcing constraints. However, to our knowledge, there have not been any studies of this. An important question is how to treat the two sets of data, training and virtual, using these approaches.

\subsubsection{Spectral Expansion Approximation}

The approach of Section 7.1 for boundary condition constraints can also be used for reduced rank GPR (Hensman et al., 2017; Solin and Särkkä, 2019). An expansion of a covariance kernel in terms of the eigenvalues of the Laplacian with periodic boundary values in an artificial box containing the data is used to approximate the covariance kernel, as in Eq. (44). The error of approximation should be small if the boundaries of the box are sufficiently far from the data 
locations. With $m$ basis functions in expansion (44), formula (45) implies that inverses are required only of matrices of size $m$. Therefore, inversion scales as $O\left(\mathrm{~m}^{3}\right)$, while multiplication for inference in Eq. (45) scales as $O\left(\mathrm{~m}^{2} \mathrm{~N}\right)$. Moreover, formulas (46) and (47), and the fact that $\Phi$ does not depend on the kernel hyperparameters, imply that the same reduced-rank advantage is present in hyperparameter training via MLE.

\subsubsection{Linear Algebra Techniques}

Rather than trying to reduce the effective size of the training and virtual constraint points, it is possible to simply approximate covariance matrix inversion using more general numerical linear algebra techniques. We expect such methods to extend more readily to constrained GPs than the methods of Section 8.3.1, although they may be less optimal and may not inform the placement of virtual constraint points.

The pseudo-inverse is often used to avoid the small eigenvalues that can corrupt predictions (Brunton and Kutz, 2019), although the singular value decomposition or eigenvalue decomposition are both computationally expensive as well. Hierarchical matrices are an efficient way of approximating the full covariance matrix in a manner amenable to fast inversion (Pouransari et al., 2017). Sub-blocks of the matrix are replaced with fixed-rank approximations, using a computational tree to organize the hierarchy of the matrix, and operations on the full matrix such as multiplication or inversion can be accomplished efficiently by operating on each of the individual "leaves" of the tree. Geoga et al. (2019) applied hierarchical matrices methods to the maximum likelihood estimation for Gaussian processes.

An alternative to inverting the covariance matrix is to set up an optimization problem for a matrix such that the error between the product of the matrix with the covariance matrix and the identity matrix is minimized (Zhao and Liu, 2013). The solution to this linear program is, of course, the precision matrix, but by adding an $L_{1}$ penalty term on the entries of the matrix to the objective function as in the least absolute shrinkage and selection operator (LASSO) regression, sparsity will be induced in the result. This estimator is referred to as constrained L1-minimization for inverse matrix estimation (CLIME) (Cai et al., 2011).

Another popular approach for modeling sparsity in random processes is to use a Gaussian Markov random field (GMRF) (Rue and Tjelmeland, 2002; Sørbye and Rue, 2014). In a GMRF, the data may be seen as forming an undirected graph where points close to each other in dataspace are connected by an edge, and points far from each other are not. Thus while all points are correlated with each other, most are only conditionally dependent on each other; this translates to a sparse precision matrix where the only off-diagonal entries correspond to points that are connected to each other. Different choices of the covariance widths or kernels, such as truncated or tapered kernels (Kaufman et al., 2008; Shaby and Ruppert, 2012), yield different levels of sparsity in the final precision matrix.

\subsubsection{Hierarchical Decomposition for Non-Gaussian Likelihoods}

Constraints that rely on non-Gaussian likelihood were reviewed in Sections 4.1.2 and 5.1. The recent work of Flaxman et al. (2015) focuses on scalable inference with non-Gaussian likelihoods on dense multidimensional grids. The key assumption enabling the use of Kronecker formulas is that the inputs $X$ are on a multidimensional Cartesian grid

$$
X=X_{1} \otimes X_{2} \otimes \ldots \otimes X_{d}
$$

Volume 1, Issue 2, 2020 
and the GP kernel in Eq. (3) is formed as a product of kernels across input dimensions

$$
K(X, X)=K_{1}\left(X_{1}\right) \otimes K_{2}\left(X_{2}\right) \otimes \ldots \otimes K_{d}\left(X_{d}\right)
$$

Under these conditions the storage requirements are reduced from $O\left(N^{2}\right)$ to $O\left(d N^{2 / d}\right)$, and the complexity of inversion is reduced from $O\left(N^{3}\right)$ to $O\left(d N^{(d+1) / d}\right)$, where $N$ is the cardinality of the full tensor grid, i.e., the number of data points, and $N^{1 / d}$ is the number of input points in each dimension.

We review the key Kronecker algebra results, including efficient matrix-vector multiplication and eigendecomposition. For matrix-vector operations

$$
(A \otimes B) X=\operatorname{vec}\left(B X A^{\top}\right)
$$

where $v=\operatorname{vec}(V)$ converts column-major formatted matrices to vectors. For higher dimensions, the expression above is applied recursively. In this approach, the full matrix is never formed, and individual steps rely only on operations with individual kernels $K_{i}$. To compute the inverse $\left[K(X, X)+\sigma^{2} I\right]^{-1}$ in Eq. (8), we use the eigendecomposition for each kernel $K_{i}=Q_{i}^{\top} \Lambda_{i} Q_{i}$, which results in

$$
K+\sigma^{2} I=\left(Q_{1}^{\top} \otimes Q_{2}^{\top} \otimes \ldots \otimes Q_{d}^{\top}\right)\left(\Lambda_{1} \otimes \Lambda_{2} \otimes \ldots \otimes \Lambda_{d}+\sigma^{2} I\right)\left(Q_{1} \otimes Q_{2} \otimes \ldots \otimes Q_{d}\right) .
$$

The inverse is evaluated as

$$
\left(K+\sigma^{2} I\right)^{-1}=\left(Q_{1}^{\top} \otimes Q_{2}^{\top} \otimes \ldots \otimes Q_{d}^{\top}\right)\left(\Lambda_{1} \otimes \Lambda_{2} \otimes \ldots \otimes \Lambda_{d}+\sigma^{2} I\right)^{-1}\left(Q_{1} \otimes Q_{2} \otimes \ldots \otimes Q_{d}\right)
$$

In this framework, the inverse of the full matrix now consists of eigendecompositions of smaller matrices.

\section{CONCLUSION}

Interest in machine learning for scientific applications has intensified in recent years, in part due to advances in algorithms, data storage, and computational analysis capabilities (Baker et al., 2019; Stevens et al., 2020). Fundamental challenges still remain when developing and using a machine learning model for scientific applications, which must satisfy physical principles. Enforcing such principles as constraints helps ensure the behavior of the model is consistent with prior physical knowledge when queried in an extrapolatory region. In other words, in addition to supplementing limited or expensive scientific data, constraints help improve the generalizability of the model in ways that simply increasing dataset size may not. Many approaches have been developed to perform physics-informed machine learning. In this survey, we have focused on constraint implementation in Gaussian processes, which are popular as machine-learned metamodels or emulators for a computational simulation.

Our survey focused on several important classes of constraints for Gaussian processes. These included positivity or bound constraints on the Gaussian processes in Section 4. When positivity constraints are applied to the derivatives of a Gaussian process, they lead to monotonicity and convexity constraints as in Section 5. This is a special example of regression with a linear transformation of a Gaussian process, which is the basis of Gaussian processes constrained by linear differential equations reviewed in Section 6. We discuss boundary value constrained Gaussian processes in Section 7. Throughout, we see that constraints can be enforced in an implicit way 
through data that satisfies the constraint, by construction of a tailored sample space, by derivation of a constrained covariance kernel, or by modifying the output or likelihood of the Gaussian process. The constraints may be enforced in a global sense, at a finite set of virtual or auxiliary points, or only in an approximate sense. We have pointed to these aspects as key features distinguishing the constraints in this survey.

Constraints introduce new practical challenges into the GPR framework. These include the analytical construction of sample spaces, transformations, or covariance kernels that inherently provide constraints; the sampling of truncated multivariate normals or intractable posterior distributions that arise when using non-Gaussian likelihoods; increased data and covariance matrix size when enforcing constraints with virtual data that leads to expanded four-block covariance; calculation of eigenvalues/eigenfunctions in bounded domains with complex geometry; the placement of virtual points or construction of spline grids in higher dimensions; and maximum likelihood training (optimization) of the hyperparameters of constrained Gaussian processes. Numerical issues are the focus of Section 8. In that section, we have also reviewed established numerical strategies for accelerating GPR. Some of these techniques have been applied to constrained Gaussian processes in the literature, while others have not. In general, the adaptation of computational strategies to constrained GPR is a relatively new field, and best practices have not yet been established. Moreover, while several codebases have been developed for constrained GPR, such GP Stuff for non-Gaussian likelihoods (Vanhatalo et al., 2013) and the li neqGPR package (López-Lopera, 2018) for the spline approach including constrained MLE, constraints have not made their way into the most widely used production codes for GPR. Furthering these computational aspects of constrained GPR remains a promising area for future work.

The field of constrained Gaussian processes has made significant advances over the past decade, and we expect significant development to continue. The purpose of this survey, while nonexhaustive, has been to catalog and investigate some of the more common approaches, guide the practitioner to identify which strategies are most appropriate for his or her needs, and point out the new computational challenges of constrained Gaussian processes and how they can be approached.

\section{ACKNOWLEDGMENTS}

This work was completed with funding granted under Sandia's Laboratory Directed Research and Development program. Sandia National Laboratories is a multimission laboratory managed and operated by National Technology and Engineering Solutions of Sandia, LLC, a wholly owned subsidiary of Honeywell International Inc. for the U.S. Department of Energy's National Nuclear Security Administration under contract DE-NA0003525. This paper describes objective technical results and analysis. Any subjective views or opinions that might be expressed in the paper do not necessarily represent the views of the U.S. Department of Energy or the United States Government.

\section{REFERENCES}

Agrell, C., Gaussian Processes with Linear Operator Inequality Constraints, 2019. arXiv:1901.03134

Albert, C.G. and Rath, K., Gaussian Process Regression for Data Fulfilling Linear Differential Equations with Localized Sources, Entropy, vol. 22, no. 152, 2020.

Bachoc, F., Lagnoux, A., López-Lopera, A.F., Maximum Likelihood Estimation for Gaussian Processes under Inequality Constraints, Electron. J. Stat., vol. 13, no. 2, pp. 2921-2969, 2019.

Volume 1, Issue 2, 2020 
Baker, N., Alexander, F., Bremer, T., Hagberg, A., Kevrekidis, Y., Najm, H., Parashar, M., Patra, A., Sethian, J., Wild, S., Willcox, K., and Lee, S., Workshop Report on Basic Research Needs for Scientific Machine Learning: Core Technologies for Artificial Intelligence, Tech. Rep., USDOE Office of Science (SC), Washington, D.C., USA, 2019.

Baldassarre, L., Rosasco, L., Barla, A., and Verri, A., Vector Field Learning via Spectral Filtering, Joint European Conf. on Machine Learning and Knowledge Discovery in Databases, Springer, pp. 56-71, 2010.

Berkeley Institute for Data Science, Physics in Machine Learning Workshop, accessed May 26, 2020, from https://bids.berkeley.edu/events/physics-machine-learning-workshop, 2019.

Berlinet, A. and Thomas-Agnan, C., Reproducing Kernel Hilbert Spaces in Probability and Statistics, Berlin: Springer Science \& Business Media, 2011.

Botev, Z.I., The Normal Law under Linear Restrictions: Simulation and Estimation via Minimax Tilting, $J$. R. Stat. Soc.: Ser. B (Stat. Method.), vol. 79, no. 1, pp. 125-148, 2017.

Brezger, A. and Steiner, W.J., Monotonic Regression based on Bayesian P-Splines: An Application to Estimating Price Response Functions from Store-Level Scanner Data, J. Bus. Econ. Stat., vol. 26, no. 1, pp. 90-104, 2008.

Brunton, S.L. and Kutz, J.N., Data-Driven Science and Engineering: Machine Learning, Dynamical Systems, and Control, Cambridge, UK: Cambridge University Press, 2019.

Brunton, S.L., Proctor, J.L., and Kutz, J.N., Discovering Governing Equations from Data by Sparse Identification of Nonlinear Dynamical Systems, Proc. Nat. Acad. Sci., vol. 113, no. 15, pp. 3932-3937, 2016.

Cai, T., Liu, W., and Luo, X., A Constrained $\ell_{1}$ Minimization Approach to Sparse Precision Matrix Estimation, J. Am. Stat. Assoc., vol. 106, no. 494, pp. 594-607, 2011.

Chilès, J.P. and Desassis, N., Fifty Years of Kriging, Handbook of Mathematical Geosciences, Berlin: Springer, pp. 589-612, 2018.

Cyr, E.C., Gulian, M.A., Patel, R.G., Perego, M., and Trask, N.A., Robust Training and Initialization of Deep Neural Networks: An Adaptive Basis Viewpoint, 2019. arXiv:1912.04862

Da Veiga, S. and Marrel, A., Gaussian Process Modeling with Inequality Constraints, Annales Faculté Sci. Toulouse, vol. 21, pp. 529-555, 2012.

Dean, J., Corrado, G., Monga, R., Chen, K., Devin, M., Mao, M., Ranzato, M., Senior, A., Tucker, P., and Yang, K., Large Scale Distributed Deep Networks, Adv. Neural Inf. Proc. Syst., pp. 1223-1231, 2012.

Driscoll, M.F., The Reproducing Kernel Hilbert Space Structure of the Sample Paths of a Gaussian Process, Prob. Theor. Related Fields, vol. 26, no. 4, pp. 309-316, 1973.

Duvenaud, D., The Kernel Cookbook: Advice on Covariance Functions, accessed 26 May, 2020, from https://www.cs.toronto.edu/ duvenaud/cookbook/, 2014.

Flaxman, S., Wilson, A., Neill, D., Nickisch, H., and Smola, A., Fast Kronecker Inference in Gaussian Processes with Non-Gaussian Likelihoods, Proc. of the 32nd International Conference on Machine Learning, F. Bach and D. Blei, Eds., Lille, France, pp. 607-616, 2015. http://proceedings.mlr.press/v37/flaxman15.html

Frankel, A., Jones, R., and Swiler, L., Tensor Basis Gaussian Process Models of Hyperelastic Materials, 2019a. arXiv:1912.10872

Frankel, A., Tachida, K., and Jones, R., Prediction of the Evolution of the Stress Field of Polycrystals Undergoing Elastic-Plastic Deformation with a Hybrid Neural Network Model, 2019b. arXiv:1910.03172

Frankel, A.L., Jones, R.E., Alleman, C., and Templeton, J.A., Predicting the Mechanical Response of Oligocrystals with Deep Learning, 2019c. arXiv:1901.10669

Fuselier Jr., E.J., Refined Error Estimates for Matrix-Valued Radial Basis Functions, PhD, Texas A\&M University, 2007. 
Genz, A. and Bretz, F., Computation of Multivariate Normal and t Probabilities, Berlin: Springer Science \& Business Media, vol. 195, 2009.

Geoga, C.J., Anitescu, M., and Stein, M.L., Scalable Gaussian Process Computations Using Hierarchical Matrices, J. Comput. Graph. Stat., pp. 1-11, 2019.

Graepel, T., Solving Noisy Linear Operator Equations by Gaussian Processes: Application to Ordinary and Partial Differential Equations, ICML, pp. 234-241, 2003.

Gramacy, R., LaGP: Large-Scale Spatial Modeling via Local Approximate Gaussian Processes in R, J. Stat. Software, Articles, vol. 72, no. 1, pp. 1-46, 2016. https://www.jstatsoft.org/v072/i01

Gramacy, R.B., Surrogates: Gaussian Process Modeling, Design, and Optimization for the Applied Sciences, Boca Raton, FL: CRC Press, 2020.

Gramacy, R.B. and Apley, D.W., Local Gaussian Process Approximation for Large Computer Experiments, J. Comput. Graph. Stat., vol. 24, no. 2, pp. 561-578, 2015.

Gulian, M., Raissi, M., Perdikaris, P., and Karniadakis, G.E., Machine Learning of Space-Fractional Differential Equations, SIAM J. Sci. Comput., vol. 41, no. 4, pp. A2485-A2509, 2019.

Hastie, T., Tibshirani, R., and Friedman, J., The Elements of Statistical Learning: Data Mining, Inference and Prediction, 2nd Ed., Berlin: Springer, 2016.

Hensman, J., Durrande, N., and Solin, A., Variational Fourier Features for Gaussian Processes, J. Mach. Learn. Res., vol. 18, no. 1, pp. 5537-5588, 2017.

Jensen, B.S., Nielsen, J.B., and Larsen, J., Bounded Gaussian Process Regression, 2013 IEEE Int. Workshop on Machine Learning for Signal Processing (MLSP), IEEE, pp. 1-6, 2013.

Jidling, C., Strain Field Modelling Using Gaussian Processes, PhD, Uppsala University, 2017.

Jidling, C., Wahlström, N., Wills, A., and Schön, T.B., Linearly Constrained Gaussian Processes, Adv. Neural Inf. Proc. Syst., pp. 1215-1224, 2017.

Jones, R., Templeton, J.A., Sanders, C.M., and Ostien, J.T., Machine Learning Models of Plastic Flow based on Representation Theory, Comput. Model. Eng. Sci., pp. 309-342, 2018.

Kanagawa, M., Hennig, P., Sejdinovic, D., and Sriperumbudur, B.K., Gaussian Processes and Kernel Methods: A Review on Connections and Equivalences, 2018. arXiv:1807.02582

Karpatne, A., Atluri, G., Faghmous, J.H., Steinbach, M., Banerjee, A., Ganguly, A., Shekhar, S., Samatova, N., and Kumar, V., Theory-Guided Data Science: A New Paradigm for Scientific Discovery from Data, IEEE Transact. Knowl. Data Eng., vol. 29, no. 10, pp. 2318-2331, 2017.

Kaufman, C.G., Schervish, M.J., and Nychka, D.W., Covariance Tapering for Likelihood-Based Estimation in Large Spatial Data Sets, J. Am. Stat. Assoc., vol. 103, no. 484, pp. 1545-1555, 2008.

Kelly, C. and Rice, J., Monotone Smoothing with Application to Dose-Response Curves and the Assessment of Synergism, Biometrics, pp. 1071-1085, 1990.

Lee, K. and Carlberg, K., Model Reduction of Dynamical Systems on Nonlinear Manifolds Using Deep Convolutional Autoencoders, 2018. arXiv: 1812.08373

Ling, J., Jones, R., and Templeton, J., Machine Learning Strategies for Systems with Invariance Properties, J. Comput. Phys., vol. 318, pp. 22-35, 2016.

López-Lopera, A.F., LineqGPR: Gaussian Process Regression Models with Linear Inequality Constraints, accessed May 26, 2020, from https://github.com/anfelopera/lineqGPR, 2018.

López-Lopera, A.F., Bachoc, F., Durrande, N., and Roustant, O., Finite-Dimensional Gaussian Approximation with Linear Inequality Constraints, SIAM/ASA J. Uncertainty Quant., vol. 6, no. 3, pp. 1224-1255, 2018.

Los Alamos Center for Nonlinear Studies, 3rd Physics Informed Machine Learning Conference, accessed May 26, 2020, from https://cnls.lanl.gov/External/workshops.php, 2020.

Volume 1, Issue 2, 2020 
Lusch, B., Kutz, J.N., and Brunton, S.L., Deep Learning for Universal Linear Embeddings of Nonlinear Dynamics, Nat. Commun., vol. 9, no. 1, p. 4950, 2018. https://www.nature.com/articles/s41467-01807210-0/

Maatouk, H., Finite-Dimensional Approximation of Gaussian Processes with Inequality Constraints, 2017. arXiv: 1706.02178

Maatouk, H. and Bay, X., A New Rejection Sampling Method for Truncated Multivariate Gaussian Random Variables Restricted to Convex Sets, Monte Carlo and Quasi-Monte Carlo Methods, Berlin: Springer, pp. 521-530, 2016.

Maatouk, H. and Bay, X., Gaussian Process Emulators for Computer Experiments with Inequality Constraints, Math. Geosci., vol. 49, no. 5, pp. 557-582, 2017.

Macêdo, I. and Castro, R., Learning Divergence-Free and Curl-Free Vector Fields with Matrix-Valued Kernels, Tech. rep., Instituto Nacional de Matematica Pura e Aplicada, 2008.

Magiera, J., Ray, D., Hesthaven, J.S., and Rohde, C., Constraint-Aware Neural Networks for Riemann Problems, 2019. arXiv:1904.12794

Mao, Z., Jagtap, A.D., and Karniadakis, G.E., Physics-Informed Neural Networks for High-Speed Flows, Comput. Methods Appl. Mech. Eng., vol. 360, p. 112789, 2020.

Microsoft, Physics $\cap$ ML: Physics Meets Machine Learning, accessed May 26, 2020, from https://www.microsoft.com/en-us/research/event/physics-ml-workshop/, 2019.

Murphy, K.P., Machine Learning: A Probabilistic Perspective, Cambridge, MA: MIT press, 2012.

Narcowich, F.J. and Ward, J.D., Generalized Hermite Interpolation via Matrix-Valued Conditionally Positive Definite Functions, Math. Comput., vol. 63, no. 208, pp. 661-687, 1994.

Pan, S. and Duraisamy, K., Data-Driven Discovery of Closure Models, SIAM J. Appl. Dyn. Syst., vol. 17, no. 4, pp. 2381-2413, 2018. https://doi.org/10.1137/18M1177263

Pensoneault, A., Yang, X., and Zhu, X., Nonnegativity-Enforced Gaussian Process Regression, 2020. arXiv: 2004.04632

Pouransari, H., Coulier, P., and Darve, E., Fast Hierarchical Solvers for Sparse Matrices Using Extended Sparsification and Low-Rank Approximation, SIAM J. Sci. Comput., vol. 39, no. 3, pp. A797-A830, 2017.

Quiñonero-Candela, J. and Rasmussen, C.E., Analysis of Some Methods for Reduced Rank Gaussian Process Regression, Switching and Learning in Feedback Systems, Berlin: Springer, pp. 98-127, 2005a.

Quiñonero-Candela, J. and Rasmussen, C.E., A Unifying View of Sparse Approximate Gaussian Process Regression, J. Mach. Learn. Res., vol. 6, pp. 1939-1959, 2005 b.

Raissi, M., Deep Hidden Physics Models: Deep Learning of Nonlinear Partial Differential Equations, $J$. Mach. Learn. Res., vol. 19, no. 1, pp. 932-955, 2018.

Raissi, M. and Karniadakis, G.E., Hidden Physics Models: Machine Learning of Nonlinear Partial Differential Equations, J. Comput. Phys., vol. 357, pp. 125-141, 2018.

Raissi, M., Perdikaris, P., and Karniadakis, G.E., Machine Learning of Linear Differential Equations Using Gaussian Processes, J. Comput. Phys., vol. 348, pp. 683-693, 2017.

Raissi, M., Perdikaris, P., and Karniadakis, G.E., Numerical Gaussian Processes for Time-Dependent and Nonlinear Partial Differential Equations, SIAM J. Sci. Comput., vol. 40, no. 1, pp. A172-A198, 2018.

Raissi, M., Perdikaris, P., and Karniadakis, G.E., Physics-Informed Neural Networks: A Deep Learning Framework for Solving Forward and Inverse Problems Involving Nonlinear Partial Differential Equations, J. Comput. Phys., vol. 378, pp. 686-707, 2019. 
Rasmussen, C.E. and Williams, C.K., Gaussian Processes for Machine Learning, Cambridge, MA: MIT Press, 2006.

Ray, P., Pati, D., and Bhattacharya, A., Efficient Bayesian Shape-Restricted Function Estimation with Constrained Gaussian Process Priors, 2019. arXiv:1902.04701

Rider, C. and Simmons, J.E., Eds., Chemical Mixtures and Combined Chemical and Non-Chemical Stressors, Ch. 15, Berlin: Springer, 2018.

Riihimäki, J. and Vehtari, A., Gaussian Processes with Monotonicity Information, Proc. of the Thirteenth International Conference on Artificial Intelligence and Statistics, pp. 645-652, 2010.

Rue, H. and Tjelmeland, H., Fitting Gaussian Markov Random Fields to Gaussian Fields, Scand. J. Stat., vol. 29, no. 1, pp. 31-49, 2002.

Sacks, J., Welch, W.J., Mitchell, T.J., and Wynn, H.P., Design and Analysis of Computer Experiments, Stat. Sci., pp. 409-423, 1989.

Salzmann, M. and Urtasun, R., Implicitly Constrained Gaussian Process Regression for Monocular NonRigid Pose Estimation, Adv. Neural Inf. Proc. Syst., pp. 2065-2073, 2010.

Santner, T.J., Williams, B.J., and Notz, W.I., The Design and Analysis of Computer Experiments, Berlin: Springer Series in Statistics, 2003.

Särkkä, S., Linear Operators and Stochastic Partial Differential Equations in Gaussian Process Regression, Int. Conf. on Artificial Neural Networks, Springer, pp. 151-158, 2011.

Seeger, M., Gaussian Processes for Machine Learning, Int. J. Neural Syst., vol. 14, no. 02, pp. 69-106, 2004.

Shaby, B. and Ruppert, D., Tapered Covariance: Bayesian Estimation and Asymptotics, J. Comput. Graph. Stat., vol. 21, no. 2, pp. 433-452, 2012.

Snelson, E., Ghahramani, Z., and Rasmussen, C.E., Warped Gaussian Processes, Adv. Neural Inf. Proc. Syst., pp. 337-344, 2004.

Solak, E., Murray-Smith, R., Leithead, W.E., Leith, D.J., and Rasmussen, C.E., Derivative Observations in Gaussian Process Models of Dynamic Systems, Adv. Neural Inf. Proc. Syst., pp. 1057-1064, 2003.

Solin, A. and Kok, M., Know Your Boundaries: Constraining Gaussian Processes by Variational Harmonic Features, Proceedings of Machine Learning Research, K. Chaudhuri and M. Sugiyama, Eds., Vol. 89 of Proc. Mach. Learn. Res., PMLR, pp. 2193-2202, 2019.

Solin, A., Kok, M., Wahlström, N., Schön, T.B., and Särkkä, S., Modeling and Interpolation of the Ambient Magnetic Field by Gaussian Processes, IEEE Transact. Rob., vol. 34, no. 4, pp. 1112-1127, 2018.

Solin, A. and Särkkä, S., Hilbert Space Methods for Reduced-Rank Gaussian Process Regression, Stat. Comput., 2019.

Song, F., Xu, C., and Karniadakis, G.E., Computing Fractional Laplacians on Complex-Geometry Domains: Algorithms and Simulations, SIAM J. Sci. Comput., vol. 39, no. 4, pp. A1320-A1344, 2017.

Sørbye, S.H. and Rue, H., Scaling Intrinsic Gaussian Markov Random Field Priors in Spatial Modelling, Spatial Stat., vol. 8, pp. 39-51, 2014.

Stanford University, Combining Artificial Intelligence and Machine Learning with Physical Sciences, accessed May 26, 2020, from https://sites.google.com/view/aaai-mlps, 2020.

Stevens, R., Taylor, V., Nichols, J., Maccabe, A.B., Yelick, K., and Brown, D., AI for Science, Tech. Rep., Argonne National Lab. (ANL), Argonne, IL (United States), 2020.

University of Washington, Physics Informed Machine Learning Workshop, accessed May 26, 2020, from http://www.databookuw.com/page-5/, 2019.

Volume 1, Issue 2, 2020 
Vanhatalo, J., Riihimäki, J., Hartikainen, J., Jylänki, P., Tolvanen, V., and Vehtari, A., GPStuff: Bayesian Modeling with Gaussian Processes, J. Mach. Learn. Res., vol. 14, no. Apr, pp. 1175-1179, 2013.

Wahlström, N., Modeling of Magnetic Fields and Extended Objects for Localization Applications, PhD, Linköping University, 2015.

Wahlström, N., Kok, M., Schön, T.B., and Gustafsson, F., Modeling Magnetic Fields Using Gaussian Processes, 2013 IEEE International Conference on Acoustics, Speech and Signal Processing, IEEE, pp. 3522-3526, 2013.

Wang, X. and Berger, J.O., Estimating Shape Constrained Functions Using Gaussian Processes, SIAM/ASA J. Uncertainty Quant., vol. 4, no. 1, pp. 1-25, 2016.

Wilson, A. and Nickisch, H., Kernel Interpolation for Scalable Structured Gaussian Processes (KISS-GP), Int. Conf. on Machine Learning, pp. 1775-1784, 2015.

Yang, X., Barajas-Solano, D., Tartakovsky, G., and Tartakovsky, A.M., Physics-Informed CoKriging: A Gaussian-Process-Regression-Based Multifidelity Method for Data-Model Convergence, J. Comput. Phys., vol. 395, pp. 410-431, 2019.

Yang, X., Tartakovsky, G., and Tartakovsky, A., Physics-Informed Kriging: A Physics-Informed Gaussian Process Regression Method for Data-Model Convergence, 2018. arXiv: 1809.03461

Zhao, T. and Liu, H., Sparse Precision Matrix Estimation with Calibration, Proc. of the 26th International Conference on Neural Information Processing Systems, pp. 2274-2282, 2013. 